\title{
Volatolomics of Sardinian and Spanish Bituminaria: Characterization of Different Accessions Using Chemometrics
}

\author{
Marianna Usai ${ }^{1, *}$, Mauro Marchetti ${ }^{2}$, Rita A.M. Melis ${ }^{3}$ and Claudio Porqueddu ${ }^{3}$ \\ 1 Department of Chemistry and Pharmacy, University of Sassari, via Muroni 23/a, 07100 Sassari, Italy \\ 2 Institute of Biomolecular Chemistry, National Research Council (CNR), Trav. La Crucca 3, 07100 Sassari, Italy; \\ mauro.marchetti@cnr.it \\ 3 Institute for the Animal Production System in the Mediterranean Environment (CNR), Traversa La Crucca 3, \\ 07040 Sassari, Italy; rita.melis@cspm.ss.cnr.it (R.A.M.M.); claudio.porqueddu@cspm.ss.cnr.it (C.P.) \\ * Correspondence: dsfusai@uniss.it; Tel.: +39-079228751
}

Citation: Usai, M.; Marchetti, M.;

Melis, R.A.; Porqueddu, C.

Volatolomics of Sardinian and Spanish Bituminaria: Characterization of Different Accessions Using Chemometrics. Molecules 2021, 26, 5247. https://doi.org/10.3390/ molecules 26175247

Academic Editor: Riccardo Petrelli

Received: 14 July 2021

Accepted: 23 August 2021

Published: 30 August 2021

Publisher's Note: MDPI stays neutral with regard to jurisdictional claims in published maps and institutional affiliations.

Copyright: (c) 2021 by the authors. Licensee MDPI, Basel, Switzerland. This article is an open access article distributed under the terms and conditions of the Creative Commons Attribution (CC BY) license (https:// creativecommons.org/licenses/by/ $4.0 /)$.

\begin{abstract}
The present study aims to determine the volatile compositions of 15 different accessions of native Sardinian populations of Bituminaria morisiana (Pignatti \& Metlesics) Greuter, Bituminaria bituminosa (L.) C. H. Stirt. (B. b.), and Spanish native accessions of B. bituminosa. Furthermore, we particularly focused on the essential oil characterization of these accessions and discriminated within populations with low furocoumarin content useful for fodder production in Mediterranean environments or furocoumarin extraction for pharmaceutical utilization. The plant extracts were analyzed by GC/MS, showing great variability in the content and composition. No differences were found in Bituminaria bituminosa (L.) C.H. Stirt. var. bituminosa essential oils, while the varieties Bituminaria bituminosa (L.) C.H. Stirt. var. crassiuscula P. Méndez, Fern. Galván \& A. Santos and Bituminaria bituminosa (L.) C.H. Stirt. var. albomarginata P. Méndez, Fern. Galván \& A. Santos are characterized by the presence of a high concentration of long-chain alcohols and of salicylic acid benzylic ester. In B. bituminosa var. albomarginata, we observed a different profile with predominance of a large concentration of alcohols as dodecanol and tetradecanol. The endemic B. morisiana can be identified for the predominant presence of farnesene. In methanolic fractions, we detected the presence of maltol, methyl citrate, methyl cumarate, santonin, and methyl linoleate. B. morisiana showed a low content of psoralens, and the accession of B. morisiana, from Siliqua indicated the presence of apocynin.
\end{abstract}

Keywords: Bituminaria genus; Bituminaria morisiana; essential oils; chemometrics tool; furocoumarins

\section{Introduction}

Bituminaria genus (Fabaceae) was first established by Linnaeus in the second edition of the Linnaeus Gen. Plant., 358 (I742) and credited to Royen. In the first edition of the Sp. Plant., 762 (1753), eight species are described, only one of which is credited to America, and it is probably an introduced plant from the Island of Madeira [1].

The morphology of the genus Bituminaria Heist. ex Fabricius (Psoraleeae, Fabaceae) is very heterogeneous and it is widespread across the Mediterranean region and Macaronesian Islands, where it is estimated to have diverged from other Psoraleoid genera approximately 6.78 million years ago. The causes of diversification can be attributed to various factors, such as habitat modifications and reproductive biology. These speciation processes on the Bituminaria genus led to eight distinct species: B. bituminosa (L.) C.H. Stirt., B. morisiana (Pignatti \& Metlesics) Greuter, B. flaccida (Nábělek) Greuter, B. basaltica Miniss., C. Brullo, Brullo, Giusso \& Sciandr., B. kyreniae Giusso, C. Brullo, Brullo, Cambria \& Miniss., B. palaestina (Bassi) Brullo, C. Brullo, Miniss., Salmeri \& Giusso, B. plumosa (Rchb.) Bogdanović, C. Brullo, Brullo, Ljubičić \& Giusso, and B. acaulis (Steven ex M. Bieb.) C.H. Stirt. [2]. 
In Europe, only Bituminaria bituminosa (L.) C.H. Stirt. var. bituminosa is present, which is a perennial legume from the Mediterranean Region and is, therefore, also able to resist drought and a hot climate. Bituminaria grows in the Mediterranean basin and Macaronesia, and it has a potential as pasture fodder for ruminants in semiarid environments, but only few selected ecotypes are cultivated in Canary Islands and Morocco for this purpose [3].

On Sardinia Island, two species of Bituminaria can be found in natural stands: B. bituminosa var. bituminosa and the endemic Bituminaria morisiana (Pignatti \& Metlesics) Greuter. They can be easily distinguished on the basis of some morphological traits and the presence of a typical bitumen smell in B. bituminosa [4]. In Spain and the Canary Islands, B. bituminosa is spontaneously grown, which exhibits a large diversity with three botanical varieties Bituminaria bituminosa var. albomarginata (albo tedera), Bituminaria bituminosa var. crassiuscula (teide tedera), and Bituminaria bituminosa var. bituminosa (tedera). This genus also grows across the Mediterranean basin, and it is known as Arabian pea or pitch trefoil $[3,5,6]$. Both species have an appreciable content of furocoumarins, compounds that are related to a low palatability of fresh fodder [7], but that can be used in therapy combining the oral drug psoralen and high-intensity longwave ultraviolet light (PUVA) [8].

Only few studies have been carried out on the furocoumarin content of Psoralea genus and Bituminaria genus, mostly on Psoralea corylifolia L. [9-12]. On B. bituminosa and its varieties, the literature is very scarce [13-17].

As far as the knowledge on the composition of the essential oil of Bituminaria genus is concerned, the paper of Bandeira et al. described the chemical composition of essential oil of Bituminaria basaltica Miniss., C. Brullo, Brullo, Giusso \& Sciandr. [17], whereas other authors have investigated essential oils in Psoralea drupacea Bunge and P. corylifolia L. In these species, the presence of bakuchiol, angelicin, $\alpha$-pinene, and limonene [18] and their antimicrobial activities $[19,20]$ were documented. Volatile composition was also assessed in different organs of B. bituminosa collected in Tuscany [21]. Another study on a B. bituminosa germplasm collected in Italy was performed on the volatile composition of fresh leaves and flowers collected at the experimental field of the University of Tuscia, Viterbo [22]. El-Seedi et al. investigated the essential oil of Psoralea pubescence (Miq.) Standl in plants collected at the Reserva Forestal ENDESA (Ecuador) [23]. A study on B. bituminosa var. bituminosa essential oil growing wild in Elba Island was published in 2016 [14].

Bituminaria genus is widely present in Sardinia, along with the endemic B. morisiana, which is very interesting for its metabolites. Various articles on B. morisiana have revealed the presence of pterocarpans [24]; in particular, a paper on B. morisiana characterized two new pterocarpans and demonstrated a low cytotoxic activity of erybraedin $C$ on all used cell lines, while erybraedin $C$ induced necrosis in leukemia Jukart T cells [25].

Recently, 15 accessions of Bituminaria: B. morisiana (Sardinian endemism), B. bituminosa, and B. bituminosa var. albomarginata, crassiuscula and bituminosa, of Sardinian and Spanish origin, were studied for their forage yield, furocoumarins, and essential oil content $[26,27]$. Furocoumarin extracts from B. morisiana were also tested for their mutagenic potential on river buffalo blood cells [28].

In this view, we conducted a comparative analysis among native Sardinian populations of B. morisiana and B. bituminosa and Spanish native accessions of $B$. bituminosa and some of its varieties. The analyses of EOs of all studied plant species were examined using chemometric tools (PCA). The aim was to characterize the volatile compounds discriminating the population of these accessions to be used in the health or herbal field. In this way, it we had the possibility to divide accessions with low furocoumarin content to use for fodder production in Mediterranean environments and accessions with high furocoumarin content useful as pharmaceutical preparations.

\section{Results}

\subsection{Chemical Composition of the Essential Oil Content}

We analyzed 15 different accessions of plants belonging to the Bituminaria genus and growing in experimental fields of the Interdepartmental Center for the Conservation and 
Enhancement of Plant Biodiversity of Sassari University, coming from different areas of Sardinia and Spain. From the aerial part of fresh plants, we extracted and characterized the essential oils and the furocoumarins.

All the obtained essential oils were diluted at the same concentration and subjected to GC and GC/MS characterization; considering all the species, 96 constituents were identified. In the six B. bituminosa var. bituminosa samples, three collected in Sardinia and three collected in Spain, the identified components were never lower than $91.45 \%$ (Table 1).

In the essential oil obtained from the accessions of B. bituminosa var. bituminosa coming from the Sardinian populations, we identified 63 different compounds, which accounted for $98.39 \%, 97.30 \%$, and $94.34 \%$ of constituents.

In the three populations native to Sardinia, among all constituents, 22 had a concentration higher than $1 \%$. In Loculi, the major constituent was caryophyllene oxide $(19.56 \%)$. $\beta$-Caryophyllene was the major constituent of Sassari station (17.30\%). Whereas germacrene $\mathrm{D}$ was the more important constituent in the accession coming from Siniscola $(10.46 \%)$, it had a minimum level in Loculi $(5.38 \%)$.

An interesting aspect concerning the composition of these essential oils was the presence of long-chain alcohols such as caryophylla-4(12),8(13)-dien-5 $\alpha$-ol, which was present only in concentrations lower than $1 \%$, caryophylla-4(14),8(15)-dien-5 $\beta$-ol, found in three stations with concentrations ranging between $2.30 \%$ and $1.44 \%$, (cis,cis)-9,12-octadecadien1-ol, present in good concentration (5.28\%) in Sassari and in Loculi (3.87\%), reaching the minimum concentration (1.85\%) in Siniscola, and (cis,cis,cis)-9,12,15-octadecatrien-1-ol, reaching a very similar concentration at all Sardinian stations (4-5\%). Another aspect was the high concentration of oxides.

In B. bituminosa var. bituminosa, derived from Spanish seeds or seedlings (58 identified constituents), the main constituents differed in each sample. In LIano del Beal, the main constituent was $\beta$-caryophyllene $(34.47 \%)$; the other well-represented components were cis$\beta$-farnesene $(8.19 \%)$ and $\alpha$-humulene $(7.75 \%)$. In Calnegre samples, the main component was cis,cis-9,12-octadecadien-1-ol (15.76\%). In San Cristòbal de la laguna samples, we found a different distribution of principal constituents; the main components were (cis,cis)-9,12octadecadien-1-ol (11.06\%), $\beta$-caryophyllene (8.18\%), (cis,cis,cis)-9,12,15-octadecatrien-1-ol $(6.58 \%)$, and caryophyllene oxide $(5.58 \%)$.

In samples from accessions of $B$. bituminosa var. albomarginata (Canary Islands) (Table 2), we identified 47 constituents representing about $93.8 \%$ of the total and a different composition between the two stations was found.

In B. $b$. albomarginata accessions coming from Caleta de Famara, the main constituents were long-chain alcohols (cis,cis)-9,12-octadecadien-1-ol (14.96\%) and (cis,cis,cis)-9,12,15octadecatrien-1-ol (9.86\%). In B. b. albomarginata accessions coming from Arecife, an original profile was found. In fact, there was a predominance of lauryl alcohol (13.83\%) (not present in Caleta de Famara) and tetradecanol (10.93\%). 
Table 1. Chemical composition of Bituminaria bituminosa (L.) C.H. Stirt. essential oils from Sardinia and Spain genotypes grown in a Sardinian experimental field.

\begin{tabular}{|c|c|c|c|c|c|c|c|c|c|c|}
\hline \multirow[b]{2}{*}{$\begin{array}{l}\text { RI Lett } \\
\text { Apolar }\end{array}$} & \multirow[b]{2}{*}{$\begin{array}{l}\text { RI Exp } \\
\text { Apolar }\end{array}$} & \multirow[b]{2}{*}{ Compounds } & \multicolumn{3}{|c|}{$\begin{array}{c}\text { Bituminaria bituminosa var. } \\
\text { bituminosa Sardinia }\end{array}$} & \multicolumn{3}{|c|}{ Bituminaria bituminosa var. bituminosa Spain } & \multirow[b]{2}{*}{ ID * } & \multirow[b]{2}{*}{ References } \\
\hline & & & $\begin{array}{l}\text { B. } b . \\
\text { Siniscola }\end{array}$ & B. b. Loculi & B.b.SS & $\begin{array}{c}B . b . \\
\text { Llano del Beal }\end{array}$ & B. b. Calnegre & $\begin{array}{c}\text { B. } b . \\
\text { San Cristòbal } \\
\text { de la Laguna }\end{array}$ & & \\
\hline 921 & 927 & tricyclene & & & & & & & Std & \\
\hline 939 & 937 & $\alpha$-pinene & & & & & & & Std & \\
\hline 955 & 954 & camphene & & & & & & & Std & \\
\hline 992 & 991 & $\beta$-myrcene & & & & & & & Std & \\
\hline 1031 & 1029 & limonene & & & & & & & Std & \\
\hline 1031 & 1030 & $\beta$-fellandrene & & & & & & & Std & \\
\hline 1052 & 1050 & trans- $\beta$-ocimene & & $0.41 \pm 0.05$ & & $1.04 \pm 0.09$ & & & Std & \\
\hline 1282 & 1275 & citronellyl formate & & $0.28 \pm 0.02$ & $0.21 \pm 0.01$ & $1.07 \pm 0.10$ & & & MS-RI & [29] \\
\hline 1320 & 1317 & 2,4-dodecadienal & & & & & & & MS-RI & \\
\hline 1323 & 1326 & methyl geraniate & & & & & & & MS-RI & \\
\hline 1345 & 1351 & $\alpha$-cubebene & $0.26 \pm 0.01$ & $0.26 \pm 0.02$ & $0.21 \pm 0.01$ & $0.58 \pm 0.04$ & & & Std & \\
\hline 1374 & 1377 & $\alpha$-copaene & $0.97 \pm 0.02$ & $0.64 \pm 0.02$ & $0.56 \pm 0.03$ & & $0.55 \pm 0.04$ & $3.15 \pm 0.22$ & Std & \\
\hline 1385 & 1385 & trans- $\beta$-damascenone & $0.23 \pm 0.01$ & $0.43 \pm 0.02$ & $0.54 \pm 0.02$ & $0.32 \pm 0.02$ & $0.22 \pm 0.01$ & $0.63 \pm 0.4$ & MS-RI & {$[30]$} \\
\hline 1387 & 1387 & $\beta$-bourbonene & $1.68 \pm 0.09$ & $1.64 \pm 0.07$ & $1.34 \pm 0.08$ & $0.51 \pm 0.02$ & $0.70 \pm 0.02$ & $1.94 \pm 0.22$ & Std & \\
\hline 1388 & 1388 & farnesene isomer & & & & & & & MS & \\
\hline 1387 & 1390 & $\beta$-cubebene & $0.38 \pm 0.02$ & & & & & & Std & \\
\hline 1389 & 1393 & $\beta$-elemene & $0.42 \pm 0.02$ & & & $0.34 \pm 0.01$ & $0.43 \pm 0.02$ & $0.74 \pm 0.07$ & Std & \\
\hline 1396 & 1396 & 1,5,8-trimethyl-1,2-dihydronaphthalene & & $0.60 \pm 0.04$ & $0.31 \pm 0.04$ & & & & MS & \\
\hline 1411 & 1409 & isocaryophyllene & & & & & & & MS-RI & [31] \\
\hline 1412 & 1412 & 1,2-dihydro-1,4,6-trimethylnaphthalene & & $0.44 \pm 0.03$ & $0.63 \pm 0.04$ & & & & MS & \\
\hline 1418 & 1418 & dihydrodehydro- $\beta$-ionone & & $0.72 \pm 0.06$ & & & $0.40 \pm 0.02$ & & MS & \\
\hline 1419 & 1419 & $\beta$-caryophyllene & $15.8 \pm 0.32$ & $18.96 \pm 0.37$ & $17.31 \pm 0.33$ & $34.47 \pm 0.62$ & $14.23 \pm 0.35$ & $8.18 \pm 0.34$ & Std & \\
\hline 1419 & 1419 & $\beta$-cedrene & $1.55 \pm 0.07$ & $1.49 \pm 0.09$ & & $0.64 \pm 0.05$ & & & Std & \\
\hline 1430 & 1433 & epi-bicyclosesquiphellandrene & & & $1.94 \pm 0.011$ & & $0.95 \pm 0.12$ & $1.50 \pm 0.05$ & MS-RI & [32] \\
\hline
\end{tabular}


Table 1. Cont.

\begin{tabular}{|c|c|c|c|c|c|c|c|c|c|c|}
\hline \multirow[b]{2}{*}{$\begin{array}{l}\text { RI Lett } \\
\text { Apolar }\end{array}$} & \multirow[b]{2}{*}{$\begin{array}{l}\text { RI Exp } \\
\text { Apolar }\end{array}$} & \multirow[b]{2}{*}{ Compounds } & \multicolumn{3}{|c|}{$\begin{array}{l}\text { Bituminaria bituminosa var. } \\
\text { bituminosa Sardinia }\end{array}$} & \multicolumn{3}{|c|}{ Bituminaria bituminosa var. bituminosa Spain } & \multirow[b]{2}{*}{ ID * } & \multirow[b]{2}{*}{ References } \\
\hline & & & $\begin{array}{c}\text { B. } b . \\
\text { Siniscola }\end{array}$ & B. $b$. Loculi & B. b. SS & $\begin{array}{c}B . b . \\
\text { Llano del Beal }\end{array}$ & B. $b$. Calnegre & $\begin{array}{c}\text { B. } b . \\
\text { San Cristòbal } \\
\text { de la Laguna }\end{array}$ & & \\
\hline 1431 & 1433 & $\beta$-gurjunene & $0.33 \pm 0.01$ & $0.39 \pm 0.03$ & $0.27 \pm 0.01$ & $0.15 \pm 0.01$ & & $0.31 \pm 0.04$ & Std & \\
\hline 1439 & 1441 & aromadendrene & & & & & & & Std & \\
\hline 1434 & 1437 & $\gamma$-elemene & & & & & & & Std & \\
\hline 1452 & 1451 & $\alpha$-humulene & $3.36 \pm 0.09$ & $3.83 \pm 0.11$ & $4.11 \pm 0.14$ & $7.75 \pm 0.13$ & $3.83 \pm 0.18$ & $2.75 \pm 0.09$ & Std & \\
\hline 1452 & 1452 & $\alpha$-himachalene & & & & & & & MS-RI & \\
\hline 1458 & 1460 & alloaromadendrene & $1.47 \pm 0.04$ & $0.90 \pm 0.05$ & $1.04 \pm 0.09$ & $0.58 \pm 0.04$ & $0.80 \pm 0.05$ & $2.66 \pm 0.14$ & MS-RI & \\
\hline 1473 & 1471 & lauryl alcohol & & & $0.25 \pm 0.01$ & & & & MS-RI & [33] \\
\hline 1478 & 1480 & $\gamma$-muurolene & $2.16 \pm 0.09$ & $1.87 \pm 0.21$ & $1.69 \pm 0.19$ & $0.69 \pm 0.04$ & $1.51 \pm 0.04$ & $2.37 \pm 0.08$ & Std & \\
\hline 1484 & 1485 & germacrene D & $10.46 \pm 0.28$ & $5.38 \pm 0.35$ & $8.62 \pm 0.27$ & $2.2 \pm 0.08$ & $4.56 \pm 0.12$ & $5.48 \pm 0.31$ & Std & \\
\hline 1493 & 1493 & epi-cubebol & $1.2 \pm 0.05$ & $0.93 \pm 0.12$ & $0.93 \pm 0.09$ & & $0.68 \pm 0.07$ & $1.14 \pm 0.07$ & MS-RI & \\
\hline 1500 & 1500 & $\alpha$-muurolene & $1.30 \pm 0.04$ & $0.98 \pm 0.09$ & $1.07 \pm 0.12$ & & $0.52 \pm 0.04$ & $1.36 \pm 0.07$ & Std & \\
\hline 1505 & 1509 & $\beta$-bisabolene & & & $0.10 \pm 0.01$ & & $0.11 \pm 0.02$ & & Std & \\
\hline 1508 & 1510 & $\alpha$-farnesene & $0.51 \pm 0.03$ & $0.31 \pm 0.02$ & & & & & Std & \\
\hline 1513 & 1514 & $\gamma$-cadinene & $0.70 \pm 0.04$ & $0.78 \pm 0.03$ & $0.65 \pm 0.02$ & & $0.31 \pm 0.02$ & $0.55 \pm 0.04$ & Std & \\
\hline 1514 & 1519 & cubebol & $0.69 \pm 0.07$ & $0.45 \pm 0.02$ & $0.64 \pm 0.02$ & & & $1.26 \pm 0.11$ & MS-RI & \\
\hline 1520 & 1522 & 7-epi- $\alpha$-selinene & & & & & & & MS-RI & \\
\hline 1521 & 1523 & $\beta$-sesquiphellandrene & & & & & & & MS-RI & \\
\hline 1522 & 1523 & $\delta$-cadinene & $2.10 \pm 0.11$ & $1.82 \pm 0.14$ & $1.76 \pm 0.08$ & $0.79 \pm 0.02$ & $1.08 \pm 0.08$ & $3.42 \pm 0.31$ & Std & \\
\hline 1539 & 1539 & $\alpha$-copaen-11-ol & & & & & & & MS-RI & \\
\hline 1548 & 1550 & elemol & & & & & & & Std & \\
\hline 1559 & 1561 & germacrene B & & & & & & & MS-RI & \\
\hline 1565 & 1564 & cis-nerolidol & $3.74 \pm 0.15$ & $0.33 \pm 0.04$ & $0.41 \pm 0.02$ & $0.44 \pm 0.01$ & $0.84 \pm 0.05$ & $0.32 \pm 0.02$ & Std & \\
\hline 1569 & 1567 & cis-3-hexenyl benzoate & $0.48 \pm 0.04$ & $0.24 \pm 0.02$ & $0.25 \pm 0.01$ & $0.53 \pm 0.04$ & & & MS-RI & [34] \\
\hline
\end{tabular}


Table 1. Cont.

\begin{tabular}{|c|c|c|c|c|c|c|c|c|c|c|}
\hline \multirow[b]{2}{*}{$\begin{array}{l}\text { RI Lett } \\
\text { Apolar }\end{array}$} & \multirow[b]{2}{*}{$\begin{array}{l}\text { RI Exp } \\
\text { Apolar }\end{array}$} & \multirow[b]{2}{*}{ Compounds } & \multicolumn{3}{|c|}{$\begin{array}{l}\text { Bituminaria bituminosa var. } \\
\text { bituminosa Sardinia }\end{array}$} & \multicolumn{3}{|c|}{ Bituminaria bituminosa var. bituminosa Spain } & \multirow[b]{2}{*}{ ID * } & \multirow[b]{2}{*}{ References } \\
\hline & & & $\begin{array}{c}\text { B. } b . \\
\text { Siniscola }\end{array}$ & B. $b$. Loculi & B. b. SS & $\begin{array}{l}\text { B. } b . \\
\text { Llano del Beal }\end{array}$ & B. b. Calnegre & $\begin{array}{c}\text { B. } b . \\
\text { San Cristòbal } \\
\text { de la Laguna }\end{array}$ & & \\
\hline 1574 & 1575 & germacrene-4-ol & $0.27 \pm 0.02$ & $0.39 \pm 0.02$ & $0.24 \pm 0.01$ & & $0.23 \pm 0.01$ & $0.80 \pm 0.04$ & MS-RI & \\
\hline 1577 & 1578 & spathulenol & $0.62 \pm 0.04$ & $0.51 \pm 0.03$ & $0.62 \pm 0.04$ & & $0.17 \pm 0.01$ & $0.51 \pm 0.02$ & MS-RI & \\
\hline 1582 & 1583 & caryophyllene oxide & $13.98 \pm 0.25$ & $19.56 \pm 0.44$ & $14.24 \pm 0.37$ & $18.5 \pm 0.28$ & $7.63 \pm 0.24$ & $5.58 \pm 0.22$ & Std & \\
\hline 1590 & 1587 & globulol & $0.39 \pm 0.02$ & $0.38 \pm 0.01$ & $0.29 \pm 0.01$ & & & & Std & \\
\hline 1592 & 1593 & cedrol & & & $0.58 \pm 0.04$ & & & & MS-RI & \\
\hline 1592 & 1594 & viridiflorene & $0.64 \pm 0.03$ & & & & $0.65 \pm 0.04$ & & MS-RI & \\
\hline 1608 & 1601 & $\alpha$-humulene-epoxide II & $1.84 \pm 0.06$ & $2.63 \pm 0.09$ & $2.11 \pm 0.13$ & $2.77 \pm 0.17$ & $1.42 \pm 0.09$ & $1.29 \pm 0.11$ & MS-RI & \\
\hline 1618 & 1614 & 1,10-di-epi-cubebol & $0.27 \pm 0.01$ & & $0.33 \pm 0.01$ & & $0.22 \pm 0.01$ & & MS-RI & \\
\hline 1618 & 1619 & cis-bisabol-11-ol & $0.51 \pm 0.01$ & & & $0.56 \pm 0.04$ & & & MS-RI & \\
\hline 1620 & 1622 & muurola-4,10(14)-dien-1 $\beta$-ol & $0.29 \pm 0.02$ & $0.51 \pm 0.07$ & & $0.36 \pm 0.04$ & & & MS-RI & \\
\hline 1630 & 1630 & longifolene aldehyde & $0.44 \pm 0.03$ & & & & & & MS & \\
\hline 1630 & 1632 & $\gamma$-eudesmol & & $0.36 \pm 0.02$ & $0.41 \pm 0.02$ & & & $2.52 \pm 0.22$ & Std & \\
\hline 1631 & 1641 & caryophylla-4(12),8(13)-dien- $5 \alpha$-ol & & $0.52 \pm 0.03$ & $0.41 \pm 0.02$ & $0.57 \pm 0.04$ & $0.38 \pm 0.04$ & & MS-RI & [35] \\
\hline 1637 & 1641 & caryophylla-4(14),8(15)-dien-5 $\beta$-ol & $2.26 \pm 0.13$ & $2.30 \pm 0.11$ & $1.44 \pm 0.09$ & $1.70 \pm 0.14$ & $0.29 \pm 0.02$ & & MS-RI & [36] \\
\hline 1644 & 1646 & $\tau$-muurolol & & & & & $1.61 \pm 0.33$ & & MS-RI & \\
\hline 1645 & 1647 & cubenol & $0.63 \pm 0.04$ & $0.63 \pm 0.04$ & $0.82 \pm 0.08$ & & $0.52 \pm 0.04$ & $0.81 \pm 0.05$ & Std & \\
\hline 1652 & 1653 & $\alpha$-cadinol & $2.71 \pm 0.09$ & $2.53 \pm 0.10$ & $3.34 \pm 0.28$ & $0.58 \pm 0.04$ & $2.23 \pm 0.09$ & $3.31 \pm 0.35$ & Std & \\
\hline 1674 & 1672 & $\beta$-bisabolol & & & & & & & Std & \\
\hline 1675 & 1677 & $n$-tetradecanol & $0.61 \pm 0.04$ & $0.75 \pm 0.04$ & $0.36 \pm 0.04$ & $3.37 \pm 0.29$ & $0.31 \pm 0.02$ & $2.57 \pm 0.22$ & MS-RI & \\
\hline 1678 & 1678 & aromadendrene-oxide 2 & $1.44 \pm 0.07$ & $1.97 \pm 0.05$ & $1.25 \pm 0.06$ & $1.48 \pm 0.11$ & $0.72 \pm 0.04$ & $0.49 \pm 0.04$ & MS-RI & [37] \\
\hline 1682 & 1682 & ledene oxide II & $2.12 \pm 0.05$ & $1.37 \pm 0.07$ & $2.31 \pm 0.07$ & & $1.02 \pm 0.08$ & $1.70 \pm 0.15$ & MS-RI & [38] \\
\hline 1704 & 1704 & bisabolene oxide & & & & & & & MS-RI & \\
\hline 1718 & 1721 & cis,cis-2,6-farnesol & & & $0.47 \pm 0.04$ & & $0.94 \pm 0.05$ & & MS-RI & [39] \\
\hline
\end{tabular}


Table 1. Cont.

\begin{tabular}{|c|c|c|c|c|c|c|c|c|c|c|}
\hline \multirow[b]{2}{*}{$\begin{array}{l}\text { RI Lett } \\
\text { Apolar }\end{array}$} & \multirow[b]{2}{*}{$\begin{array}{l}\text { RI Exp } \\
\text { Apolar }\end{array}$} & \multirow[b]{2}{*}{ Compounds } & \multicolumn{3}{|c|}{$\begin{array}{l}\text { Bituminaria bituminosa var. } \\
\text { bituminosa Sardinia }\end{array}$} & \multicolumn{3}{|c|}{ Bituminaria bituminosa var. bituminosa Spain } & \multirow[b]{2}{*}{$\mathrm{ID}^{*}$} & \multirow[b]{2}{*}{ References } \\
\hline & & & $\begin{array}{l}\text { B. } b . \\
\text { Siniscola }\end{array}$ & B. b. Loculi & B.b. SS & $\begin{array}{c}\text { B. } b . \\
\text { Llano del Beal }\end{array}$ & B. b. Calnegre & $\begin{array}{c}\text { B. } b . \\
\text { San Cristòbal } \\
\text { de la Laguna }\end{array}$ & & \\
\hline 1756 & 1757 & myristic acid & & & & & & & MS-RI & \\
\hline 1760 & 1762 & benzyl benzoate & $0.19 \pm 0.01$ & $0.29 \pm 0.01$ & $0.29 \pm 0.01$ & & $0.34 \pm 0.02$ & $0.45 \pm 0.02$ & MS-RI & \\
\hline 1774 & 1781 & 1-pentadecanol & & & & & & & MS-RI & \\
\hline 1792 & 1792 & 1,2-15,16-diepoxy-hexadecane & $0.33 \pm 0.02$ & $0.25 \pm 0.01$ & & & $0.98 \pm 0.04$ & $0.77 \pm 0.04$ & MS & \\
\hline 1810 & 1816 & cis-11-hexadecenal & $0.55 \pm 0.01$ & & & & $0.90 \pm 0.04$ & $0.48 \pm 0.02$ & MS-RI & \\
\hline 1827 & 1827 & benzyl salicylate & & & & & & & MS-RI & [40] \\
\hline 1863 & 1864 & 1-hexadecanol & & & & & $1.44 \pm 0.09$ & & MS & \\
\hline 1863 & 1866 & cis-9-hexadecen-1-ol & & & & & $0.25 \pm 0.02$ & & MS-RI & \\
\hline 1890 & 1890 & 2-methylhexadecan-1-ol & $0.68 \pm 0.04$ & $0.99 \pm 0.02$ & & & & & MS & \\
\hline 1946 & 1944 & palmitic acid & $1.29 \pm 0.05$ & $1.11 \pm 0.09$ & $0.93 \pm 0.06$ & $0.59 \pm 0.04$ & $1.15 \pm 0.17$ & $2.65 \pm 0.27$ & MS-RI & \\
\hline 2046 & 2046 & geranyl linalool & & & & $0.45 \pm 0.04$ & & & MS & \\
\hline 2058 & 2058 & cis-cis-cis-9,12,15-octadecatrien-1-ol & $5.2 \pm 0.31$ & $4.97 \pm 0.044$ & $4.06 \pm 0.27$ & $0.34 \pm 0.02$ & $6.92 \pm 0.17$ & $6.58 \pm 0.20$ & MS-RI & [41] \\
\hline 2060 & 2060 & cis-9-octadecen-1-ol & & & & & & & MS-RI & \\
\hline 2074 & 2074 & n-octadecyl alcohol & & & & & & & MS-RI & \\
\hline 2104 & 2104 & 12-methyl-E,E-2,13-octadecadien-1-ol & $0.19 \pm 0.01$ & & & & & $0.47 \pm 0.04$ & MS-RI & \\
\hline 2114 & 2114 & phytol & $4.25 \pm 0.25$ & $3.02 \pm 0.33$ & $1.12 \pm 0.9$ & $1.66 \pm 0.13$ & $4.88 \pm 0.06$ & $3.95 \pm 0.25$ & MS-RI & \\
\hline 2178 & 2178 & linolenic acid & & $0.45 \pm 0.04$ & $0.23 \pm 0.01$ & $0.65 \pm 0.04$ & & $0.11 \pm 0.01$ & MS-RI & \\
\hline 2279 & 2279 & methyl 11,14,17-icosatrienoate & & $0.81 \pm 0.04$ & $0.52 \pm 0.04$ & $0.38 \pm 0.02$ & & $2.33 \pm 0.13$ & MS-RI & \\
\hline \multirow[t]{3}{*}{2241} & 2241 & $\begin{array}{l}\text { trans-trans-cis-1,3,12-nonadecatriene-5,14- } \\
\text { diol }\end{array}$ & & & & & & & MS-RI & \\
\hline & & No. of identified constituents & 49 & 48 & 49 & 34 & 44 & 40 & & \\
\hline & & & 98.09 & 96.65 & 94.03 & 95.68 & 92.76 & 91.45 & & \\
\hline
\end{tabular}

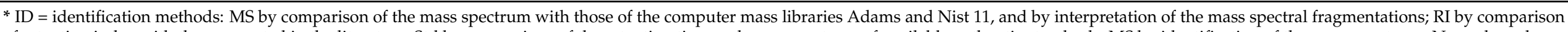

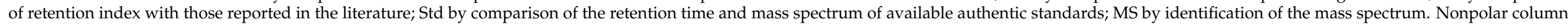
ZB-5. Data are the means of three replicates. 


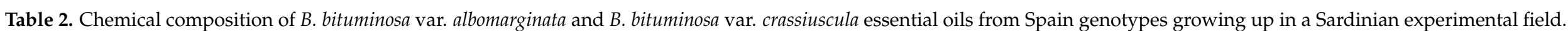

\begin{tabular}{|c|c|c|c|c|c|c|c|c|}
\hline \multirow[b]{2}{*}{$\begin{array}{l}\text { RI Lett } \\
\text { Apolar }\end{array}$} & \multirow[b]{2}{*}{$\begin{array}{l}\text { RI Exp } \\
\text { Apolar }\end{array}$} & \multirow[b]{2}{*}{ Compounds } & \multicolumn{2}{|c|}{ Bituminaria bituminosa var. albomarginata } & \multicolumn{2}{|c|}{ Bituminaria bituminosa var. crassiuscula } & \multirow[b]{2}{*}{ ID * } & \multirow[b]{2}{*}{ References } \\
\hline & & & $\begin{array}{l}\text { B. } b \text {. var. albomarginata } \\
\text { Caleta de Famara }\end{array}$ & $\begin{array}{c}\text { B. } b . \text { var. albomarginata } \\
\text { Arecife }\end{array}$ & $\begin{array}{c}\text { B. } b \text {. var. crassiuscula } \\
\text { San Cristobal de la Laguna }\end{array}$ & $\begin{array}{c}\text { B. } b . \text { var. } \\
\text { crassiuscula Vilaflor }\end{array}$ & & \\
\hline 921 & 927 & tricyclene & & & & & Std & \\
\hline 939 & 937 & $\alpha$-pinene & & & & & Std & \\
\hline 955 & 954 & camphene & & & & & Std & \\
\hline 992 & 991 & $\beta$-myrcene & & & $0.37 \pm 0.04$ & & Std & \\
\hline 1031 & 1029 & limonene & & & & & Std & \\
\hline 1031 & 1030 & $\beta$-fellandrene & & & & & Std & \\
\hline 1052 & 1050 & trans- $\beta$-ocimene & & $0.13 \pm 0.01$ & & & Std & \\
\hline 1282 & 1275 & citronellyl formate & & $0.16 \pm 0.01$ & & & MS-RI & [29] \\
\hline 1320 & 1317 & 2,4-dodecadienal & & & & $0.78 \pm 0.09$ & MS-RI & \\
\hline 1323 & 1326 & methyl geraniate & & & $0.50 \pm 0.08$ & & MS-RI & \\
\hline 1345 & 1351 & $\alpha$-cubebene & & $0.28 \pm 0.01$ & & & Std & \\
\hline 1373 & 1375 & $\alpha$-ylangene & & & & & Std & \\
\hline 1385 & 1385 & trans- $\beta$-damascenone & $0.25 \pm 0.01$ & & & $0.35 \pm 0.04$ & MS-RI & [30] \\
\hline 1387 & 1387 & $\beta$-bourbonene & $1.43 \pm 0.05$ & $0.82 \pm 0.11$ & $0.46 \pm 0.05$ & & Std & \\
\hline 1388 & 1388 & farnesene isomer & & & & & MS & \\
\hline 1387 & 1390 & $\beta$-cubebene & & & & & Std & \\
\hline 1389 & 1393 & $\beta$-elemene & $0.39 \pm 0.02$ & $0.96 \pm 0.11$ & $0.74 \pm 0.04$ & $0.44 \pm 0.04$ & Std & \\
\hline 1396 & 1396 & 1,5,8-trimethyl-1,2-dihydronaphthalene & & & & & MS & \\
\hline 1411 & 1409 & isocaryophyllene & & & & $0.42 \pm 0.04$ & MS-RI & [31] \\
\hline 1412 & 1412 & 1,2-dihydro-1,4,6-trimethylnaphthalene & & & & & MS & \\
\hline 1418 & 1418 & dihydrodehydro- $\beta$-ionone & & & & & MS & \\
\hline 1419 & 1419 & $\beta$-caryophyllene & $7.62 \pm 0.22$ & $4.32 \pm 0.24$ & $21.98 \pm 0.48$ & $24.89 \pm 0.51$ & Std & \\
\hline 1419 & 1419 & $\beta$-cedrene & $0.91 \pm 0.05$ & $1.10 \pm 0.08$ & $0.44 \pm 0.04$ & $0.53 \pm 0.04$ & Std & \\
\hline 1430 & 1433 & epi-bicyclosesquiphellandrene & & & & & MS-RI & [32] \\
\hline 1431 & 1433 & $\beta$-gurjunene & $0.40 \pm 0.02$ & & & & Std & \\
\hline 1439 & 1441 & aromadendrene & & & & & Std & \\
\hline
\end{tabular}


Table 2. Cont

\begin{tabular}{|c|c|c|c|c|c|c|c|c|}
\hline \multirow[b]{2}{*}{$\begin{array}{l}\text { RI Lett } \\
\text { Apolar }\end{array}$} & \multirow[b]{2}{*}{$\begin{array}{l}\text { RI Exp } \\
\text { Apolar }\end{array}$} & \multirow[b]{2}{*}{ Compounds } & \multicolumn{2}{|c|}{ Bituminaria bituminosa var. albomarginata } & \multicolumn{2}{|c|}{ Bituminaria bituminosa var. crassiuscula } & \multirow[b]{2}{*}{ ID * } & \multirow[b]{2}{*}{ References } \\
\hline & & & $\begin{array}{l}\text { B. } b \text {. var. albomarginata } \\
\text { Caleta de Famara }\end{array}$ & $\begin{array}{c}\text { B. } b . \text { var. albomarginata } \\
\text { Arecife }\end{array}$ & $\begin{array}{c}\text { B. } b . \text { var. crassiuscula } \\
\text { San Cristòbal de la Laguna }\end{array}$ & $\begin{array}{c}\text { B. } b . \text { var. } \\
\text { crassiuscula Vilaflor }\end{array}$ & & \\
\hline 1434 & 1437 & $\gamma$-elemene & & & $0.51 \pm 0.04$ & & Std & \\
\hline 1440 & 1443 & cis- $\beta$-farnesene & $0.61 \pm 0.04$ & $2.97 \pm 0.33$ & & $0.53 \pm 0.04$ & Std & \\
\hline 1452 & 1451 & $\alpha$-humulene & $2.97 \pm 0.17$ & $1.56 \pm 0.18$ & $7.53 \pm 0.24$ & $4.92 \pm 0.41$ & Std & \\
\hline 1452 & 1452 & $\alpha$-himachalene & & & $0.57 \pm 0.04$ & & MS-RI & \\
\hline 1458 & 1460 & alloaromadendrene & $2.16 \pm 0.12$ & & $0.99 \pm 0.06$ & $0.78 \pm 0.05$ & MS-RI & \\
\hline 1473 & 1471 & lauryl alcohol & & $13.83 \pm 0.45$ & & & MS-RI & [33] \\
\hline 1478 & 1480 & $\gamma$-muurolene & $2.07 \pm 0.09$ & $3.14 \pm 0.22$ & $1.52 \pm 0.33$ & $0.46 \pm 0.04$ & Std & \\
\hline 1479 & 1483 & $\alpha$-curcumene & & & $0.83 \pm 0.12$ & $0.21 \pm 0.02$ & MS-RI & \\
\hline 1484 & 1485 & germacrene D & $8.45 \pm 0.32$ & $12.40 \pm 0.20$ & $2.97 \pm 0.32$ & $1.72 \pm 0.33$ & Std & \\
\hline 1493 & 1493 & epi-cubebol & $1.18 \pm 0.08$ & $0.66 \pm 0.12$ & $0.81 \pm 0.08$ & $0.45 \pm 0.02$ & MS-RI & \\
\hline 1493 & 1494 & $\alpha$-zingiberene & & & & & MS-RI & \\
\hline 1500 & 1500 & $\alpha$-muurolene & $1.17 \pm 0.10$ & $2.08 \pm 0.09$ & $0.88 \pm 0.04$ & $0.23 \pm 0.01$ & Std & \\
\hline 1508 & 1510 & $\alpha$-farnesene & & & & & Std & \\
\hline 1513 & 1514 & $\gamma$-cadinene & & $0.39 \pm 0.04$ & $1.49 \pm 0.22$ & $0.21 \pm 0.01$ & Std & \\
\hline 1514 & 1519 & cubebol & $1.91 \pm 0.22$ & & $0.95 \pm 0.06$ & $0.86 \pm 0.05$ & MS-RI & \\
\hline 1520 & 1522 & 7-epi- $\alpha$-selinene & & $0.76 \pm 0.04$ & & & MS-RI & \\
\hline 1521 & 1523 & $\beta$-sesquiphellandrene & & $2.05 \pm 0.15$ & & & MS-RI & \\
\hline 1522 & 1523 & $\delta$-cadinene & $2.96 \pm 0.33$ & $2.71 \pm 0.31$ & $0.59 \pm 0.04$ & $1.22 \pm 0.21$ & Std & \\
\hline 1539 & 1539 & $\alpha$-copaen-11-ol & & $0.55 \pm 0.05$ & & $0.24 \pm 0.01$ & MS-RI & \\
\hline 1548 & 1550 & elemol & & & $0.59 \pm 0.04$ & & Std & \\
\hline 1559 & 1561 & germacrene B & $0.42 \pm 0.04$ & $0.35 \pm 0.02$ & & & MS-RI & \\
\hline 1565 & 1564 & cis-nerolidol & & $0.22 \pm 0.02$ & & & Std & \\
\hline 1569 & 1567 & cis-3-hexenyl benzoate & & & $1.12 \pm 0.11$ & & MS-RI & [34] \\
\hline 1574 & 1575 & germacrene-4-ol & & $0.63 \pm 0.04$ & & & MS-RI & \\
\hline 1577 & 1578 & spathulenol & $0.43 \pm 0.04$ & $0.44 \pm 0.04$ & & & MS-RI & \\
\hline
\end{tabular}


Table 2. Cont.

\begin{tabular}{|c|c|c|c|c|c|c|c|c|}
\hline \multirow[b]{2}{*}{$\begin{array}{l}\text { RI Lett } \\
\text { Apolar }\end{array}$} & \multirow[b]{2}{*}{$\begin{array}{l}\text { RI Exp } \\
\text { Apolar }\end{array}$} & \multirow[b]{2}{*}{ Compounds } & \multicolumn{2}{|c|}{ Bituminaria bituminosa var. albomarginata } & \multicolumn{2}{|c|}{ Bituminaria bituminosa var. crassiuscula } & \multirow[b]{2}{*}{ ID * } & \multirow[b]{2}{*}{ References } \\
\hline & & & $\begin{array}{l}\text { B. } b \text {. var. albomarginata } \\
\text { Caleta de Famara }\end{array}$ & $\begin{array}{c}\text { B. } b . \text { var. albomarginata } \\
\text { Arecife }\end{array}$ & $\begin{array}{c}\text { B. } b . \text { var. crassiuscula } \\
\text { San Cristòbal de la Laguna }\end{array}$ & $\begin{array}{c}\text { B. } b . \text { var. } \\
\text { crassiuscula Vilaflor }\end{array}$ & & \\
\hline 1582 & 1583 & caryophyllene oxide & $3.50 \pm 0.25$ & $1.44 \pm 0.11$ & $7.43 \pm 0.23$ & $11.02 \pm 0.24$ & Std & \\
\hline 1589 & 1586 & isocaryophyllene & & & & & MS-RI & \\
\hline 1590 & 1587 & globulol & & & $0.98 \pm 0.13$ & $0.75 \pm 0.07$ & Std & \\
\hline 1592 & 1593 & cedrol & & & & & MS-RI & \\
\hline 1592 & 1594 & viridiflorene & $2.37 \pm 0.19$ & $0.81 \pm 0.04$ & & & MS-RI & \\
\hline 1608 & 1601 & $\alpha$-humulene-epoxide II & $1.04 \pm 0.12$ & $0.56 \pm 0.03$ & $1.91 \pm 0.14$ & $1.35 \pm 0.33$ & MS-RI & \\
\hline 1618 & 1614 & 1,10-di-epi-cubebol & $0.95 \pm 0.08$ & $1.21 \pm 0.11$ & $0.68 \pm 0.08$ & $0.42 \pm 0.04$ & MS-RI & \\
\hline 1618 & 1619 & cis-bisabol-11-ol & & & & & MS-RI & \\
\hline 1620 & 1622 & muurola-4,10(14)-dien-1 $\beta$-ol & & & & & MS-RI & \\
\hline 1629 & 1627 & 1-epi-cubenol & & & & & MS-RI & \\
\hline 1630 & 1630 & longifolene aldehyde & & & & & MS & \\
\hline 1630 & 1632 & $\gamma$-eudesmol & & & & & Std & \\
\hline 1637 & 1641 & caryophylla-4(14),8(15)-dien-5 $\beta$-ol & & & $0.95 \pm 0.05$ & $2.20 \pm 0.17$ & MS-RI & [36] \\
\hline 1644 & 1646 & $\tau$-muurolol & & & & & MS-RI & \\
\hline 1645 & 1647 & cubenol & $0.96 \pm 0.07$ & & & & Std & \\
\hline 1652 & 1653 & $\alpha$-cadinol & $3.95 \pm 0.37$ & $3.14 \pm 0.13$ & $1.63 \pm 0.11$ & $0.94 \pm 0.09$ & Std & \\
\hline 1674 & 1672 & $\beta$-bisabolol & & & $0.32 \pm 0.02$ & & Std & \\
\hline 1675 & 1677 & $n$-tetradecanol & $0.78 \pm 0.08$ & $10.93 \pm 0.51$ & & & MS-RI & \\
\hline 1678 & 1678 & aromadendrene oxide 2 & & & $0.39 \pm 0.02$ & $1.01 \pm 0.08$ & MS-RI & [37] \\
\hline 1682 & 1682 & ledene oxide II & $2.02 \pm 0.13$ & $1.48 \pm 0.18$ & $0.68 \pm 0.04$ & $0.43 \pm 0.04$ & MS-RI & [38] \\
\hline 1704 & 1704 & bisabolene oxide & & & & & MS-RI & \\
\hline 1718 & 1721 & cis,cis-2,6-farnesol & & & & & MS-RI & [39] \\
\hline 1756 & 1757 & myristic acid & & & $0.31 \pm 0.02$ & $0.48 \pm 0.04$ & MS-RI & \\
\hline 1760 & 1762 & benzyl benzoate & $0.82 \pm 0.08$ & & $3.05 \pm 0.22$ & $2.38 \pm 0.34$ & MS-RI & \\
\hline 1774 & 1781 & 1-pentadecanol & & & & & MS-RI & \\
\hline
\end{tabular}


Table 2. Cont.

\begin{tabular}{|c|c|c|c|c|c|c|c|c|}
\hline \multirow[b]{2}{*}{$\begin{array}{l}\text { RI Lett } \\
\text { Apolar }\end{array}$} & \multirow[b]{2}{*}{$\begin{array}{l}\text { RI Exp } \\
\text { Apolar }\end{array}$} & \multirow[b]{2}{*}{ Compounds } & \multicolumn{2}{|c|}{ Bituminaria bituminosa var. albomarginata } & \multicolumn{2}{|c|}{ Bituminaria bituminosa var. crassiuscula } & \multirow[b]{2}{*}{ ID * } & \multirow[b]{2}{*}{ References } \\
\hline & & & $\begin{array}{l}\text { B. b. var. albomarginata } \\
\text { Caleta de Famara }\end{array}$ & $\begin{array}{c}\text { B. } b . \text { var. albomarginata } \\
\text { Arecife }\end{array}$ & $\begin{array}{c}\text { B. } b . \text { var. crassiuscula } \\
\text { San Cristòbal de la Laguna }\end{array}$ & $\begin{array}{c}\text { B. } b . \text { var. } \\
\text { crassiuscula Vilaflor }\end{array}$ & & \\
\hline 1792 & 1792 & 1,2-15,16-diepoxy-hexadecane & $0.73 \pm 0.04$ & $0.67 \pm 0.04$ & $0.84 \pm 0.10$ & $0.86 \pm 0.13$ & MS & \\
\hline 1810 & 1816 & cis-11-hexadecenal & $1.19 \pm 0.06$ & $0.73 \pm 0.05$ & $0.99 \pm 0.11$ & $1.01 \pm 0.07$ & MS-RI & \\
\hline 1827 & 1827 & benzyl salicylate & & & $3.45 \pm 0.31$ & $3.51 \pm 0.34$ & MS-RI & [40] \\
\hline 1863 & 1864 & 1-hexadecanol & & & & & MS & \\
\hline 1863 & 1866 & cis-9-hexadecen-1-ol & & & & & MS-RI & \\
\hline 1890 & 1890 & 2-methylhexadecan-1-ol & $1.54 \pm 0.13$ & $0.87 \pm 0.04$ & & & MS & \\
\hline 1946 & 1944 & palmitic acid & $4.23 \pm 0.22$ & $1.12 \pm 0.11$ & $2.37 \pm 0.26$ & $1.61 \pm 0.22$ & MS-RI & \\
\hline 2046 & 2046 & geranyl linalool & & & $0.58 \pm 0.04$ & & MS & \\
\hline 2052 & 2052 & cis-cis-9,12-octadecadien-1-ol & $14.96 \pm 0.40$ & $5.64 \pm 0.24$ & $11.54 \pm 0.38$ & $12.03 \pm 0.41$ & MS-RI & [22] \\
\hline 2058 & 2058 & cis-cis-cis-9,12,15-octadecatrien-1-ol & $9.86 \pm 0.21$ & $4.83 \pm 0.31$ & $6.71 \pm 0.24$ & $7.33 \pm 0.31$ & MS-RI & [41] \\
\hline 2060 & 2060 & cis-9-octadecen-1-ol & & & & & MS-RI & \\
\hline 2074 & 2074 & n-octadecyl alcohol & & & & & MS-RI & \\
\hline 2114 & 2114 & phytol & $4.89 \pm 0.19$ & $3.18 \pm 0.18$ & $2.76 \pm 0.18$ & $5.13 \pm 0.27$ & MS-RI & \\
\hline 2178 & 2178 & linolenic acid & $2.72 \pm 0.08$ & & $1.19 \pm 0.14$ & $1.14 \pm 0.12$ & MS-RI & \\
\hline 2279 & 2279 & methyl 11,14,17-icosatrienoate & & & & & MS-RI & \\
\hline \multirow[t]{3}{*}{2241} & 2241 & trans-trans-cis-1,3,12-nonadecatriene-5,14-diol & & $0.40 \pm 0.11$ & & & MS-RI & \\
\hline & & No. of identified constituents & 36 & 39 & 43 & 38 & & \\
\hline & & & 93.82 & 92.71 & 97.17 & 94.47 & & \\
\hline
\end{tabular}

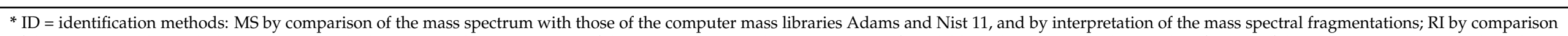

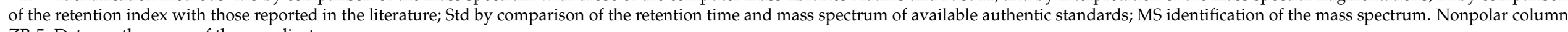
ZB-5. Data are the mean of three replicates. 
B. bituminosa var. crassiuscula belonging from Spanish native plants was characterized by the presence of 49 constituents, representing about $97 \%$ and $94 \%$ of the total. Among these constituents, 21 exceeded $1 \%$. A common feature of oils at these two stations was represented by a high concentration of $\beta$-caryophyllene (22-25\%) and caryophyllene oxide $11 \%$ in Vilaflor and about 7\% in San Cristòbal de la laguna. A component present only in these two oils was benzyl salicylate, which is a compound frequently used in cosmetics as a fragrance or UV light absorber [42]. Another constituent present in high concentration in these varieties was benzyl benzoate (3.05\% and $2.38 \%$ ), which is also used in cosmetics as fragrance but is better known for the topical treatment of human scabies [43].

In Bituminaria morisiana Pign. et Metlesics (Sardinian endemism), it was possible to identify 54 constituents of the essence in toto, representing from $93.41 \%$ to $96.75 \%$ of the total (Table 3).

Twenty-six constituents reach a concentration higher than $1 \%$. Five were present in a considerable amount. cis- $\beta$-Farnesene was the main constituent in all samples, from a minimum of $26.91 \%$ in Bitti accession to a maximum of $41.77 \%$ in Punta Giglio accession. The concentration in the other three stations varied from $34.36 \%$ to $37 \%$. Other constituents present in very high concentration were two long-chain alcohols (cis,cis)-9,12-octadecadien1-ol (linoleyl alcohol) from a minimum of $9.72 \%$ in Bitti to a maximum of $17.73 \%$ in Punta Giglio. Germacrene D was present in all samples with a high concentration in Monte Gonareddu and a minimum in Punta Giglio 2.84\%. $\beta$-Caryophyllene was well represented in the two stations of Bitti and Siliqua with concentrations of $9.92 \%$ and $9 \%$, respectively. In Bitti accession, $21.76 \%$ of alcohols deriving from fatty acids were present, as well as in Punta Giglio (22.32\%), while, in the other stations, fatty alcohols reached a concentration range between $16 \%$ and $18 \%$.

\subsection{Methanolic Extracts}

The methanolic extracts were partitioned using $\mathrm{H}_{2} \mathrm{O} / \mathrm{CHCl}_{3}$ and analyzed by GC/MS (see Section 4) [44]. From the methanolic extracts, we obtained 15 samples that were analyzed by GC and GC/MS. The content of furocoumarins is expressed in $\mathrm{mg} / 100 \mathrm{~g}$ of fresh plant (Figure 1). In the six analyzed samples of B. b. var. bituminosa, Monte Rosello showed a very high content of furocoumarins. In these samples, we found $750.2 \mathrm{mg} / \mathrm{kg}$ angelicin and $108.7 \mathrm{mg} / \mathrm{kg}$ psoralen calculated in fresh plant. In general, all samples from $B . b$. var. bituminosa showed a larger quantity of angelicin than psoralen. The analyzed extracts from B. $b$. var. bituminosa species from plants coming from Sardinia and Spain showed, in addition to the expected furocoumarin content, good amounts of maltol, trimethyl citrate, methyl coumarate, santonin, and methyl linoleate. In the crude extracts derived from our samples, we found a good concentration of maltol (e.g., $34.71 \%$ in Calnegre and $31.56 \%$ in San Cristòbal de la Laguna). In the Sardinian samples, the maximum content of maltol in the crude extract was found in the accession derived from Loculi (14.89\%). In the Spanish varieties, salicylic acid was found in the crude methanol extract, varying from $2.78 \%$ in San Cristòbal de la laguna B. b. var. bituminosa to $0.35 \%$ in LIano del Beal B. b. var. bituminosa. The other varieties from Spain showed the highest concentration of maltol in $B . b$. var. crassiuscula from Vilaflor (39.32\%), while a high concentration (33.96\%) was also found in San Cristòbal de la laguna station. 
Table 3. Chemical composition of Sardinian endemic Bituminaria morisiana (Pignatti \& Metlesics) Greuter essential oils growing up in a Sardinian experimental field.

\begin{tabular}{|c|c|c|c|c|c|c|c|c|c|}
\hline \multirow[b]{2}{*}{$\begin{array}{l}\text { RI Lett } \\
\text { Apolar }\end{array}$} & \multirow[b]{2}{*}{$\begin{array}{l}\text { RI Exp } \\
\text { Apolar }\end{array}$} & \multirow[b]{2}{*}{ Compounds } & \multicolumn{4}{|c|}{ Bituminaria Morisiana } & \multirow[b]{2}{*}{$\begin{array}{c}\text { Monte } \\
\text { Gonareddu }\end{array}$} & \multirow[b]{2}{*}{ ID * } & \multirow[b]{2}{*}{ References } \\
\hline & & & Punta Giglio & Burcei & Bitti & Siliqua & & & \\
\hline 921 & 927 & tricyclene & & & & $0.17 \pm 0.01$ & & Std & \\
\hline 939 & 937 & $\alpha$-pinene & & & & $0.67 \pm 0.02$ & & Std & \\
\hline 955 & 954 & camphene & & & & $0.39 \pm 0.01$ & & Std & \\
\hline 992 & 991 & $\beta$-myrcene & & & & $0.72 \pm 0.04$ & & Std & \\
\hline 1031 & 1030 & $\beta$-fellandrene & & & & $0.26 \pm 0.01$ & & Std & \\
\hline 1052 & 1050 & trans- $\beta$-ocimene & & & & & & Std & \\
\hline 1282 & 1275 & citronellyl formate & & & & & & MS-RI & [29] \\
\hline 1320 & 1317 & 2,4-dodecadienal & & & & & & MS-RI & \\
\hline 1323 & 1326 & methyl geraniate & & & & & & MS-RI & \\
\hline 1345 & 1351 & $\alpha$-cubebene & & $0.39 \pm 0.01$ & $0.31 \pm 0.01$ & $0.43 \pm 0.02$ & & Std & \\
\hline 1374 & 1377 & $\alpha$-copaene & $0.43 \pm 0.02$ & $0.20 \pm 0.01$ & $0.55 \pm 0.02$ & $0.35 \pm 0.02$ & $0.65 \pm 0.02$ & Std & \\
\hline 1385 & 1385 & trans- $\beta$-damascenone & & & & & & MS-RI & {$[30]$} \\
\hline 1387 & 1387 & $\beta$-bourbonene & $0.42 \pm 0.04$ & $0.84 \pm 0.04$ & $0.74 \pm 0.03$ & $0.35 \pm 0.02$ & $0.92 \pm 0.05$ & Std & \\
\hline 1388 & 1388 & farnesene isomer & $0.21 \pm 0.01$ & $0.30 \pm 0.02$ & & & & MS & \\
\hline 1387 & 1390 & $\beta$-cubebene & $0.40 \pm 0.02$ & & $0.08 \pm 0.01$ & & & Std & \\
\hline 1389 & 1393 & $\beta$-elemene & & & & & & Std & \\
\hline 1396 & 1396 & 1,5,8-trimethyl-1,2-dihydronaphthalene & & & & & & MS & \\
\hline 1411 & 1409 & isocaryophyllene & & & & & & MS-RI & [31] \\
\hline 1412 & 1412 & 1,2-dihydro-1,4,6-trimethylnaphthalene & & & & & & MS & \\
\hline 1418 & 1418 & dihydrodehydro- $\beta$-ionone & & & & & & MS & \\
\hline 1419 & 1419 & $\beta$-caryophyllene & $0.62 \pm 0.04$ & $2.91 \pm 0.11$ & $9.92 \pm 0.29$ & $9.06 \pm 0.26$ & $4.54 \pm 0.19$ & Std & \\
\hline 1419 & 1419 & $\beta$-cedrene & $1.41 \pm 0.09$ & $0.76 \pm 0.05$ & & $0.56 \pm 0.04$ & & Std & \\
\hline
\end{tabular}


Table 3. Cont.

\begin{tabular}{|c|c|c|c|c|c|c|c|c|c|}
\hline \multirow[b]{2}{*}{$\begin{array}{l}\text { RI Lett } \\
\text { Apolar }\end{array}$} & \multirow[b]{2}{*}{$\begin{array}{l}\text { RI Exp } \\
\text { Apolar }\end{array}$} & \multirow[b]{2}{*}{ Compounds } & \multicolumn{5}{|c|}{ Bituminaria Morisiana } & \multirow[b]{2}{*}{ ID * } & \multirow[b]{2}{*}{ Reference } \\
\hline & & & Punta Giglio & Burcei & Bitti & Siliqua & $\begin{array}{c}\text { Monte } \\
\text { Gonareddu }\end{array}$ & & \\
\hline 1430 & 1433 & epi-bicyclosesquiphellandrene & & & & & & MS-RI & [32] \\
\hline 1431 & 1433 & $\beta$-gurjunene & $0.61 \pm 0.04$ & & $0.80 \pm 0.04$ & & & Std & \\
\hline 1439 & 1441 & aromadendrene & & $0.18 \pm 0.01$ & $0.20 \pm 0.01$ & & & Std & \\
\hline 1434 & 1437 & $\gamma$-elemene & & & & & & Std & \\
\hline 1452 & 1451 & $\alpha$-humulene & & & & & & Std & \\
\hline 1452 & 1452 & $\alpha$-himachalene & & & & & & MS-RI & \\
\hline 1458 & 1460 & alloaromadendrene & $0.68 \pm 0.04$ & & $1.40 \pm 0.11$ & $0.84 \pm 0.06$ & $0.76 \pm 0.04$ & MS-RI & \\
\hline 1473 & 1471 & lauryl alcohol & & & & & & MS-RI & [33] \\
\hline 1478 & 1480 & $\gamma$-muurolene & $1.20 \pm 0.13$ & $2.13 \pm 0.24$ & $2.00 \pm 0.19$ & $1.35 \pm 0.07$ & $1.66 \pm 0.33$ & Std & \\
\hline 1479 & 1483 & $\alpha$-curcumene & & & & & & MS-RI & \\
\hline 1493 & 1493 & epi-cubebol & $0.94 \pm 0.11$ & $1.54 \pm 0.21$ & $1.24 \pm 0.08$ & $1.11 \pm 0.10$ & $1.38 \pm 0.14$ & MS-RI & \\
\hline 1493 & 1494 & $\alpha$-zingiberene & $2.08 \pm 0.24$ & $1.73 \pm 0.19$ & $1.50 \pm 0.06$ & $1.74 \pm 0.22$ & $1.25 \pm 0.11$ & MS-RI & \\
\hline 1500 & 1500 & $\alpha$-muurolene & $0.88 \pm 0.09$ & $1.22 \pm 0.09$ & $0.83 \pm 0.04$ & $1.29 \pm 0.16$ & $0.90 \pm 0.08$ & Std & \\
\hline 1505 & 1509 & $\beta$-bisabolene & & & & & & Std & \\
\hline 1508 & 1510 & $\alpha$-farnesene & $0.42 \pm 0.04$ & $0.41 \pm 0.04$ & $0.25 \pm 0.02$ & $0.32 \pm 0.04$ & & Std & \\
\hline 1513 & 1514 & $\gamma$-cadinene & $0.65 \pm 0.04$ & $0.95 \pm 0.08$ & $0.81 \pm 0.04$ & $0.63 \pm 0.04$ & $0.90 \pm 0.08$ & Std & \\
\hline 1514 & 1519 & cubebol & $0.57 \pm 0.04$ & $0.75 \pm 0.04$ & $0.65 \pm 0.04$ & $0.76 \pm 0.05$ & $0.76 \pm 0.04$ & MS-RI & \\
\hline 1520 & 1522 & 7-epi- $\alpha$-selinene & & & & & & MS-RI & \\
\hline 1521 & 1523 & $\beta$-sesquiphellandrene & & & & & & MS-RI & \\
\hline 1522 & 1523 & $\delta$-cadinene & $1.72 \pm 0.19$ & $2.85 \pm 0.38$ & $2.22 \pm 0.31$ & $2.27 \pm 0.25$ & $2.59 \pm 0.33$ & Std & \\
\hline 1539 & 1539 & $\alpha$-copaen-11-ol & & $0.46 \pm 0.04$ & & $0.33 \pm 0.05$ & & MS-RI & \\
\hline 1548 & 1550 & elemol & & & $0.21 \pm 0.01$ & & & Std & \\
\hline
\end{tabular}


Table 3. Cont.

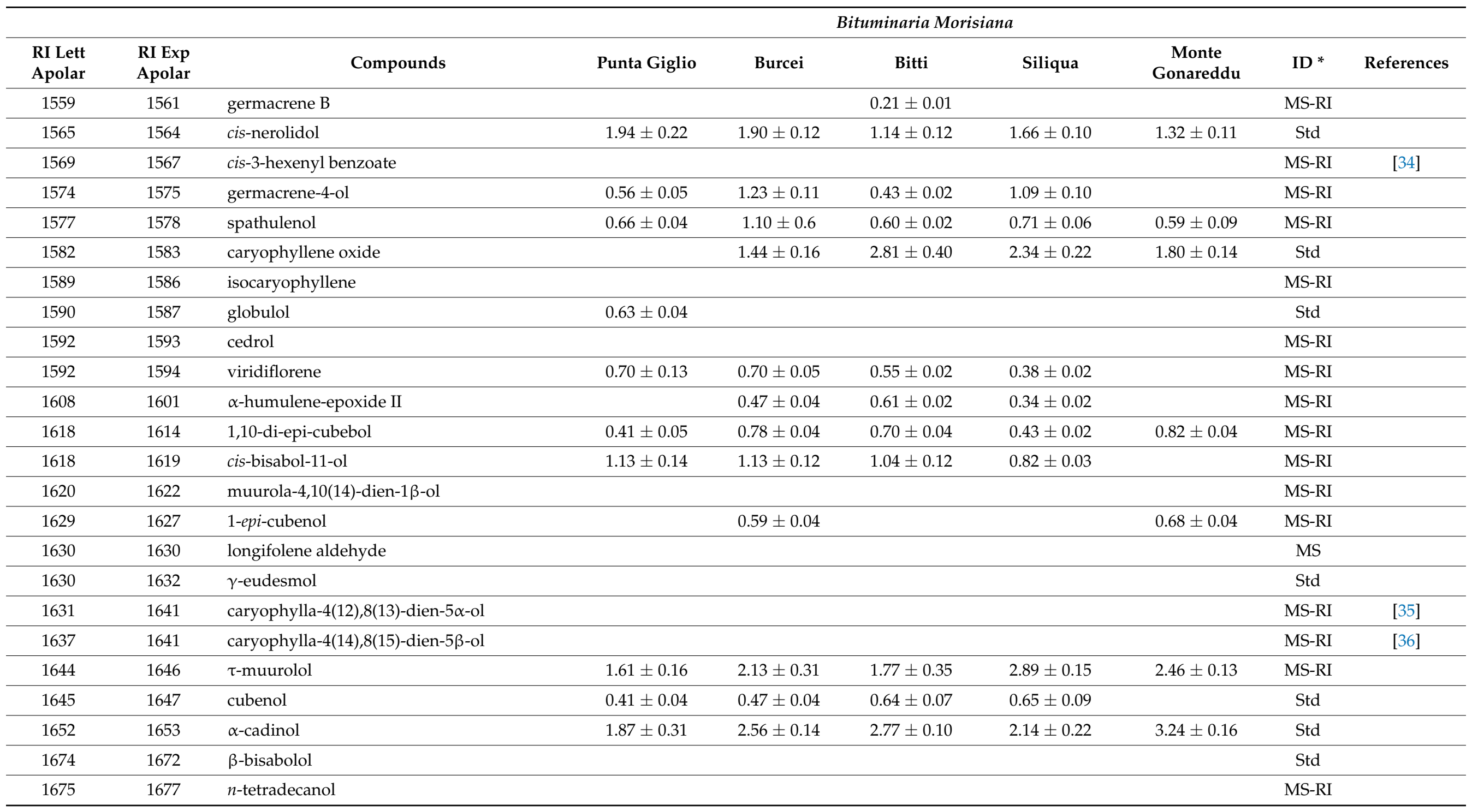


Table 3. Cont.

\begin{tabular}{|c|c|c|c|c|c|c|c|c|c|}
\hline \multirow[b]{2}{*}{$\begin{array}{l}\text { RI Lett } \\
\text { Apolar }\end{array}$} & \multirow[b]{2}{*}{$\begin{array}{l}\text { RI Exp } \\
\text { Apolar }\end{array}$} & \multirow[b]{2}{*}{ Compounds } & \multicolumn{5}{|c|}{ Bituminaria Morisiana } & \multirow[b]{2}{*}{ ID * } & \multirow[b]{2}{*}{ References } \\
\hline & & & Punta Giglio & Burcei & Bitti & Siliqua & $\begin{array}{c}\text { Monte } \\
\text { Gonareddu }\end{array}$ & & \\
\hline 1678 & 1678 & aromadendrene-oxide 2 & & & & $0.62 \pm 0.04$ & & MS-RI & [37] \\
\hline 1682 & 1682 & ledene oxide II & $0.30 \pm 0.02$ & $0.83 \pm 0.04$ & $0.87 \pm 0.04$ & & $1.10 \pm 0.10$ & MS-RI & {$[38]$} \\
\hline 1704 & 1704 & bisabolene oxide & $0.50 \pm 0.04$ & $0.71 \pm 0.04$ & $0.20 \pm 0.02$ & & & MS-RI & \\
\hline 1718 & 1721 & cis,cis-2,6-farnesol & $1.76 \pm 0.21$ & $1.26 \pm 0.09$ & $0.87 \pm 0.04$ & $1.04 \pm 0.10$ & $1.22 \pm 0.11$ & MS-RI & [39] \\
\hline 1760 & 1762 & benzyl benzoate & & & & & & MS-RI & \\
\hline 1774 & 1781 & 1-pentadecanol & & $1.14 \pm 0.11$ & & & & MS-RI & \\
\hline 1792 & 1792 & 1,2-15,16-diepoxy-hexadecane & & & & & & MS & \\
\hline 1810 & 1816 & cis-11-hexadecenal & & & & & & MS-RI & \\
\hline 1827 & 1827 & benzyl salicylate & & & & & & MS-RI & [40] \\
\hline 1863 & 1864 & 1-hexadecanol & $1.10 \pm 0.11$ & $1.14 \pm 0.07$ & $1.80 \pm 0.15$ & $1.37 \pm 0.14$ & & MS & \\
\hline 1890 & 1890 & 2-methylhexadecan-1-ol & & & & & $1.05 \pm 0.09$ & MS & \\
\hline 1946 & 1944 & palmitic acid & & & & & & MS-RI & \\
\hline 2046 & 2046 & geranyl linalool & & & & & & MS & \\
\hline 2052 & 2052 & cis-cis-9,12-octadecadien-1-ol & $17.73 \pm 0.44$ & $14.74 \pm 0.36$ & $9.72 \pm 0.22$ & $13.51 \pm 0.39$ & $15.37 \pm 0.37$ & MS-RI & [22] \\
\hline 2058 & 2058 & cis-cis-cis-9,12,15-octadecatrien-1-ol & $4.59 \pm 0.21$ & $3.58 \pm 0.27$ & $7.53 \pm 0.28$ & $3.27 \pm 0.12$ & $2.98 \pm 0.16$ & MS-RI & [41] \\
\hline 2060 & 2060 & cis-9-octadecen-1-ol & & & $4.51 \pm 0.17$ & & & MS-RI & \\
\hline 2074 & 2074 & $n$-octadecyl alcohol & $0.92 \pm 0.05$ & $0.62 \pm 0.04$ & $0.71 \pm 0.08$ & & & MS-RI & \\
\hline 2104 & 2104 & 12-methyl-E,E-2,13-octadecadien-1-ol & & & & & & MS-RI & \\
\hline 2114 & 2114 & phytol & $0.94 \pm 0.05$ & $0.73 \pm 0.04$ & $1.46 \pm 0.26$ & $0.52 \pm 0.02$ & $1.46 \pm 0.10$ & MS-RI & \\
\hline 2178 & 2178 & linolenic acid & & & & & & MS-RI & \\
\hline 2279 & 2279 & methyl 11,14,17-icosatrienoate & & & & & & MS-RI & \\
\hline
\end{tabular}


Table 3. Cont.

\begin{tabular}{|c|c|c|c|c|c|c|c|c|c|}
\hline \multirow[b]{2}{*}{$\begin{array}{l}\text { RI Lett } \\
\text { Apolar }\end{array}$} & \multirow[b]{2}{*}{$\begin{array}{l}\text { RI Exp } \\
\text { Apolar }\end{array}$} & \multirow[b]{2}{*}{ Compounds } & \multicolumn{5}{|c|}{ Bituminaria Morisiana } & \multirow[b]{2}{*}{ ID * } & \multirow[b]{2}{*}{ References } \\
\hline & & & Punta Giglio & Burcei & Bitti & Siliqua & $\begin{array}{c}\text { Monte } \\
\text { Gonareddu }\end{array}$ & & \\
\hline \multirow[t]{3}{*}{2241} & 2241 & trans-trans-cis-1,3,12-nonadecatriene-5,14-diol & & & & & & MS-RI & \\
\hline & & No. of identified constituents & 37 & 40 & 42 & 40 & 27 & & \\
\hline & & & 96.75 & 94.84 & 95.19 & 96.65 & 93.41 & & \\
\hline
\end{tabular}

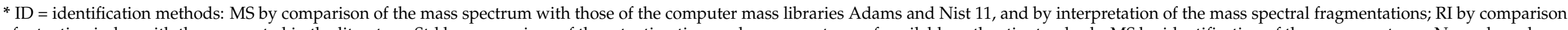

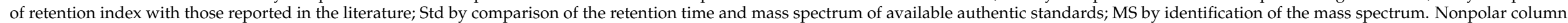
ZB-5. Data are the mean of three replicates. 


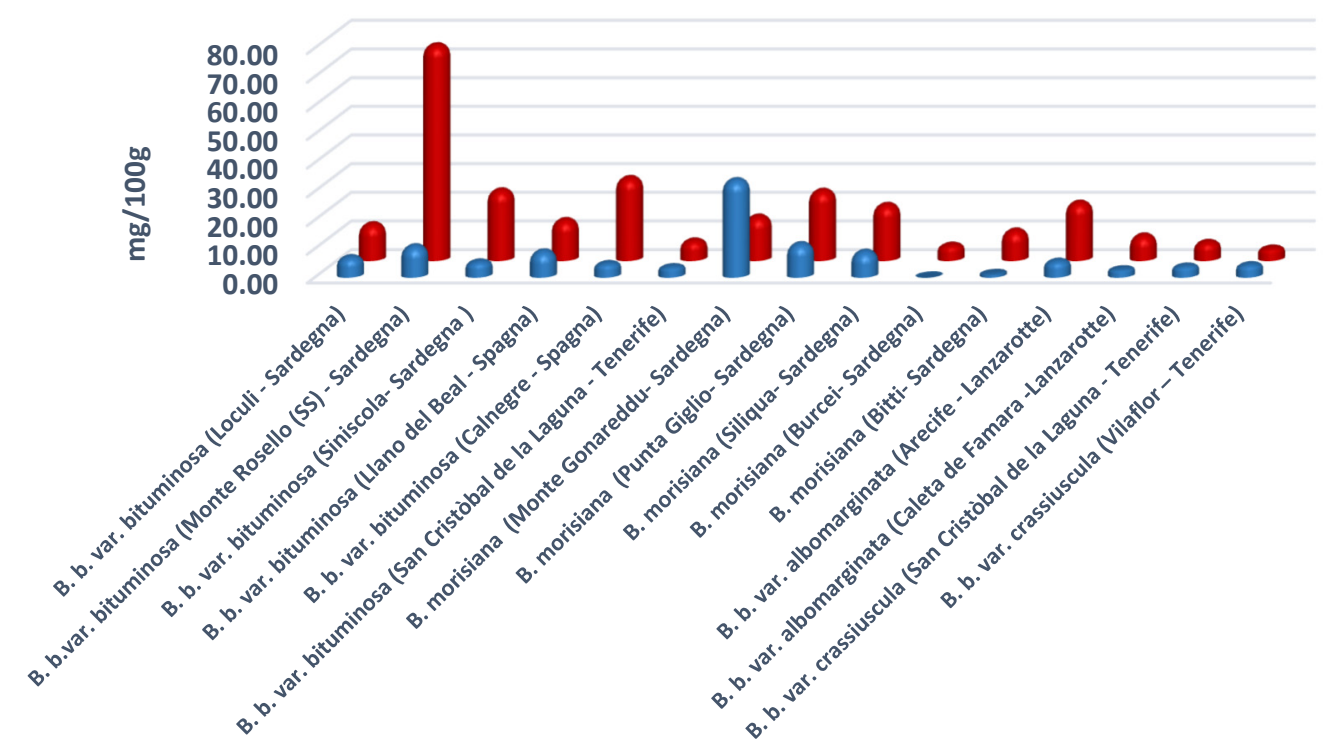

mg Psoralen on 100g of fresh plant

mg Angelicin on 100g of fresh plant

Figure 1. Content of psoralen and angelicin in the analyzed Bituminaria species and varieties (mg/100 g, fresh plant).

The endemic species of Sardinia, B. morisiana, generally showed a low content of furocoumarins, while the accessions native to Burcei did not contain more than $55 \mathrm{mg} / \mathrm{kg}$ of these substances. An exception is constituted by B. morisiana derived from the accession of Monte Gonareddu, which surprisingly had a high content of furocoumarins (psoralen $339.4 \mathrm{mg} / \mathrm{kg}$ and angelicin $154.3 \mathrm{mg} / \mathrm{kg}$ ).

In terms of the other characterized compounds, in the endemic B. morisiana, maltol reached a maximum of $19.63 \%$ in the crude extract derived from the Siliqua station accession. Only in the extract derived from B. morisiana from Siliqua was a small quantity of apocynin found $(1.54 \%)$.

\subsection{Statistical Analysis}

The obtained data were submitted to multivariate statistical evaluation to verify the biodiversity through secondary metabolites. The PCA analysis performed using only essential oil components (96 different compounds were identified) (Figure 2) clearly shows three different groups of plants; $B . b$. var. bituminosa and B. $b$. var. albomarginata were grouped, showing the homogeneity of secondary metabolites present in the essential oil. On the contrary, the accessions of B. b. var. crassiuscula and B. morisiana were welldefined groups.

Using all identified volatile metabolites coming from water distillation and methanolic extraction for PCA statistical analysis, it was possible to distinguish $B . b$. var. morisiana from $B$. bituminosa and all its varieties. Figure 3 reports the plot score of this analysis. 


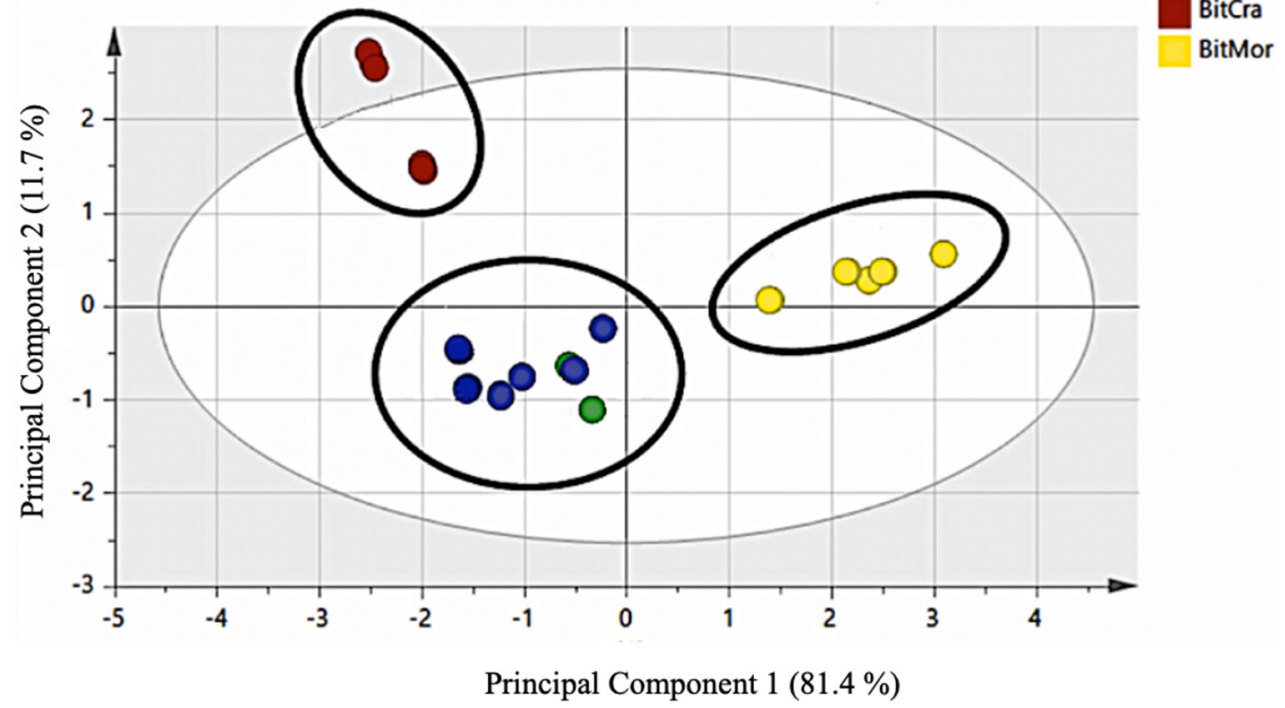

Figure 2. Score plot of PCA analysis performed using the essential oil components. Principal components 1 and 2 represented $93.1 \%$ of the variance.

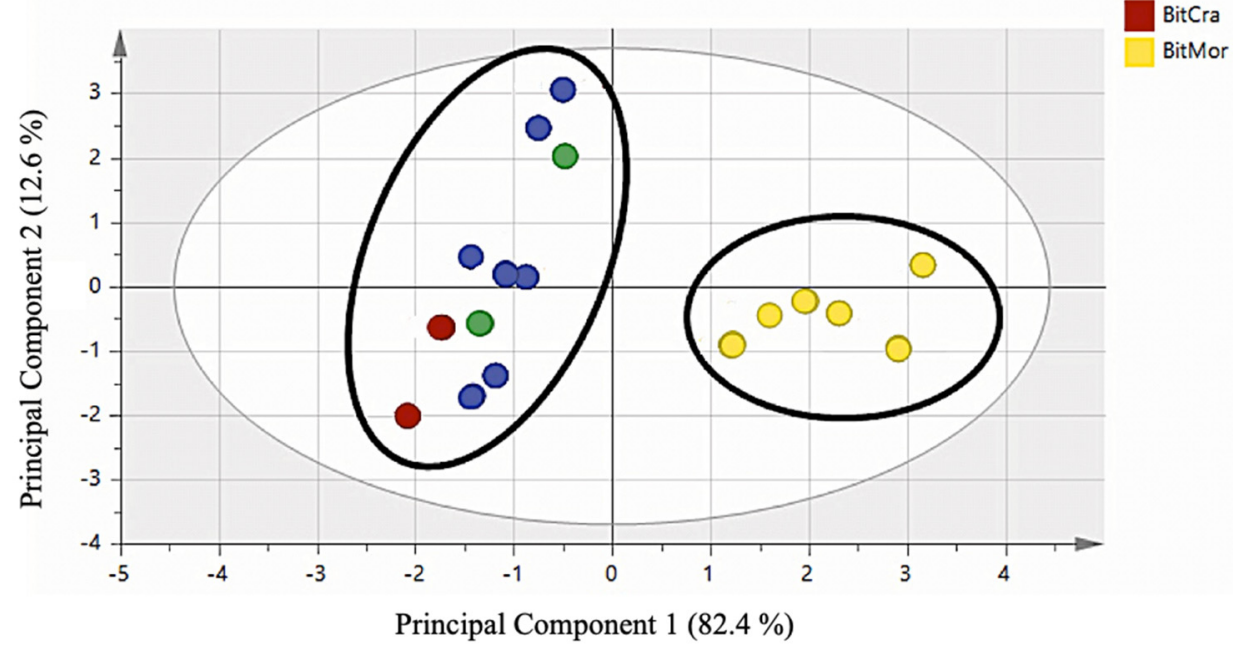

Figure 3. Score plot of PCA statistical analysis of B. morisiana and B. bituminosa varieties. Principal components 1 and 2 represented $95.0 \%$ of the variance.

\section{Discussion}

The plants we analyzed showed great variability with regard to the content and the composition of essential oils. It is not easy to identify the accessions of $B$. $b$. var. bituminosa because there are no decisively discriminating constituents. Comparing the samples coming from Sardinian accessions and that from native plants from Spain, we can note that the most represented constituents were always the same, albeit in different concentrations. A twofold higher concentration of $\beta$-caryophyllene was found in LIano del Beal compared with Sardinian samples. In other compounds, e.g., caryophyllene oxide, there were no significant differences in concentration.

Comparing $B . b$. var. albomarginata and $B . b$. var. crassiuscula, it was possible to note some differences; in $B . b$. var. albomarginata with respect to $B . b$. var. crassiuscula, we found a higher concentration of $\alpha$-copaene, germacrene $\mathrm{D}$, and palmitic acid. Moreover, in B. $b$. var. albomarginata, some compounds were present in contrast to B. $b$. var. crassiuscula, such 
as lauryl alcohol, $\beta$-sesquiphellandrene, $n$-tetradecanol, and viridiflorene. Benzyl benzoate, also present in some other varieties, reached its maximum in B. b. var. crassiuscula. B. b. var. crassiuscula also contained long-chain alcohols and benzyl salicylate.

In B. morisiana, we observed the preponderant presence of farnesene, unique to this essential oil; moreover, germacrene $\mathrm{D}$ was present in all samples with a high concentration in Monte Gonareddu and a minimum concentration in Punta Giglio. This volatile sesquiterpene belonging to the germacrene family is typically produced in plants as an antimicrobial and insecticidal or as an insect pheromone.

The concentration of essential oils distilled from Bituminaria genus was to predict their use in pharmaceutical applications, especially in terms of furanocoumarins such as psoralen and angelicin. Psoralens are widely used to treat human skin diseases and for their antimicrobial activity and anti-HIV effects. Angelicin has calmative, sedative, and anticonvulsant activities and is used for the treatment of thalassemia (US Patent No.: US2006/0111433(A1)). On this basis, we proceeded to quantify the concentration of furocoumarins and other volatile products, which are easily extracted by methanol, such as maltol, trimethyl citrate, methyl coumarate, santonin, and methyl linoleate, which might have medicinal interest. All these substances give to these plants an interest that goes beyond the simple furocoumarin content and enhance their effect. For instance, maltol itself is known for its odor of cotton candy and caramel and is used to impart a sweet aroma to fragrances. Some synthetic derivatives of maltol showed limited in vitro antiproliferative activity toward cancer cells lines [45].

Analyzing all the varieties, we found many other compounds having pharmaceutic interest. For example, in all Spanish varieties, we found salicylic acid, which is an antiinflammatory that might increase the effect of furocoumarins. In the extract derived from B. morisiana from Siliqua, we found a small quantity of apocynin, which is a very interesting compound, since it is a selective inhibitor of the phagocyte NADPH oxidase Nox2, which can be applied orally and is remarkably effective at low dose [46].

The quantified furocoumarins in our samples (Figure 1) were very significant, indicating a strong correlation between the unpleasant smell of the plant and the content of psoralens. In particular, the accessions native to Burcei did not contain more than $55 \mathrm{mg} / \mathrm{kg}$ of these substances, and it is very interesting to note that these species did not exhibit the characteristic odor of bitumen. On the contrary, other accessions contained a relatively large amount of furocoumarins; these plants could be really exploited in phytotherapy as a furocoumarin source, e.g., in the treatment of psoriasis according to the BALNEOPUVA methodology (BATH-PUVA) [47]. In this view, the fraction extracted with the methanol/acidic water solution partitioned in $\mathrm{H}_{2} \mathrm{O} / \mathrm{CHCl}_{3}$ (see Section 4) proved to be a valuable source of anti-inflammatory compounds and may be well suited for the treatment of psoriasis using the BALNEO-PUVA methodology. In particular, the accession of $B . b$. var. bituminosa from Monte Rosello would be able to easily provide the concentration of furocoumarin necessary for this health treatment.

B. b. var. albomarginata and B. $b$. var. crassiuscula derived from Spain accessions showed a low content of psoralens.

The obtained data coming from essential oil analyses or methanol extract analyses were submitted to multivariate statistical evaluation to verify the biodiversity through secondary metabolites and furnished a very interesting indication, enabling the distinction of B. morisiana from B. bituminosa and its varieties (Figure 3). Moreover, using only the essential oil components, it was possible to divide the accessions into different groups of plants. B. $b$. var. bituminosa and B. $b$. var. albomarginata were grouped together, showing the homogeneity of secondary metabolites present in their essential oils. On the contrary, the accessions B. $b$. var. crassiuscula and B. morisiana were well defined. These results are interesting if compared with those published by A. Muñoz et al. [3], where morphological and molecular analyses were used in the principal component analysis and enabled the characterization of different $B$. bituminosa accessions coming from southeast Spain and the Canary Islands into six homogenous groups. The authors reported that 
B. b. var. crassiuscula can be well differentiated from other Bituminaria plants in terms of its morphological characteristics.

\section{Materials and Methods}

\subsection{Chemicals and Instrumentation}

The fertilization of plant material was carried out using $\mathrm{P}_{2} \mathrm{O}_{5}$ (NIT Greenhouse grade $25 \mathrm{~kg}$ ) from Haifa-Italia srl (Bologna, Italy). The distilled water used for the essential oil isolation was produced in the laboratory, whereas anhydrous $\mathrm{Na}_{2} \mathrm{SO}_{4}$ was obtained from Merck S.p.A. (Sigma-Aldrich) (Milano, Italy). The quantitative GC analyses were performed using a Hewlett-Packard Model 5890A GC equipped with an automatic injector HP 7673 (now Agilent Technologies Italia S.p.A, Milano, Italy) and GC/MS analyses were conducted using an Agilent Technologies model 7820A Milano, Italy connected with a MS detector 5977E MSD (Agilent Technologies Italia S.p.A, Milano, Italy) equipped with an automatic injector HP 7673 for qualitative analyses. The carrier gas helium was obtained from SAPIO, and the columns (Phenomenex ZB-5, Torrance, CA, USA) for GC and GC/MS analyses were from ThermoFisher scientific Italia (Monza, Italy). The $400 \mathrm{MHz}$ NMR spectra were recorded using a VARIAN (Mercury plus) spectrometer (now Agilent Technologies Italia S.p.A, Milano, Italy).

\subsection{Plant Material}

An experimental field was established in CBV (Interdepartmental Center for the Conservation and Enhancement of Plant Biodiversity Center) of Sassari University located in Surigheddu district $\left(40^{\circ} 35^{\prime} 49^{\prime \prime} \mathrm{N} ; 8^{\circ} 22^{\prime} 47^{\prime \prime} \mathrm{E}\right)$ close to Alghero in northwest Sardinia. The site has a Mediterranean climate with an average annual rainfall of $540 \mathrm{~mm}$ and an alluvial soil with a high limestone content and neutral $\mathrm{pH}$ (6.9). The cultivation of different species and varieties of B. bituminosa was carried out by researchers of ISPAAM Institute (Institute for the Animal Production System in the Mediterranean Environment) of CNR (Research National Council), who handled the germplasm. They oversaw the bio-morphological screening concerning the accessions collected from different stations of Sardinia, and they took care of all agronomic factors, seed production, and its components. They also planted the seeds from the Spanish accessions. The samples of plant material derived from certified seeds or plantlets were treated after collection to avoid the degradation of biomass. The collecting stations for Sardinian B. b. var. bituminosa plants are reported in Table 4 and shown in Figure 4.

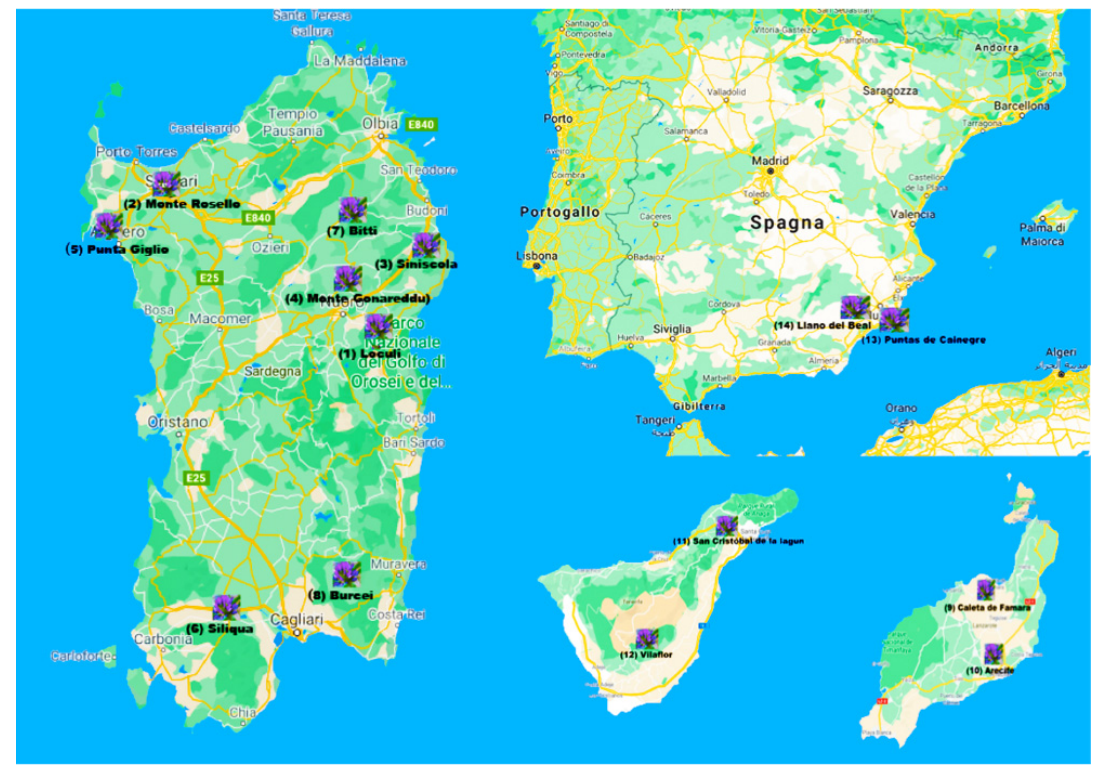

Figure 4. Collecting stations for accessions of Sardinian and Spanish Bituminaria plants. 
Table 4. Collecting stations for accessions of Sardinian and Spanish B. bituminosa (and its varieties) and endemic B. morisiana plants.

\begin{tabular}{|c|c|c|c|c|}
\hline Station $\left(\mathbf{n}^{\circ}\right)$ & Coordinates & Soil Substrate & a.s.l. & Geomorphology \\
\hline \multicolumn{5}{|c|}{ Collecting stations for Sardinian B. bituminosa var bituminosa plants } \\
\hline Loculi (1) & $\begin{array}{l}40^{\circ} 24^{\prime} 29.7^{\prime \prime} \mathrm{N} \\
09^{\circ} 36^{\prime} 25.0^{\prime \prime} \mathrm{E}\end{array}$ & granite soil & $26 \mathrm{~m}$ & flat land \\
\hline Monte Rosello (2) & $\begin{array}{l}40^{\circ} 43^{\prime} 53.8^{\prime \prime} \mathrm{N} \\
08^{\circ} 33^{\prime} 32.2^{\prime \prime} \mathrm{E}\end{array}$ & limestone & $225 \mathrm{~m}$ & hill \\
\hline Siniscola (3) & $\begin{array}{l}40^{\circ} 34^{\prime} 46.7^{\prime \prime} \mathrm{N} \\
09^{\circ} 41^{\prime} 38.4^{\prime \prime} \mathrm{E}\end{array}$ & metamorphic rocks & $38 \mathrm{~m}$ & flat land \\
\hline \multicolumn{5}{|c|}{ Collecting stations for Sardinian endemic species B. morisiana plants } \\
\hline Monte Gonareddu (4) & $\begin{array}{l}40^{\circ} 13^{\prime} 42.4^{\prime \prime} \mathrm{N} \\
09^{\circ} 11^{\prime} 53.1^{\prime \prime} \mathrm{E}\end{array}$ & limestone & $1035 \mathrm{~m}$ & mountain \\
\hline Punta Giglio (5) & $\begin{array}{l}40^{\circ} 34^{\prime} 08.4^{\prime \prime} \mathrm{N} \\
08^{\circ} 12^{\prime} 16.7^{\prime \prime} \mathrm{E}\end{array}$ & limestone & $80 \mathrm{~m}$ & promontory \\
\hline Siliqua (6) & $\begin{array}{l}39^{\circ} 15^{\prime} 07.7^{\prime \prime} \mathrm{N} \\
08^{\circ} 46^{\prime} 57.2^{\prime \prime} \mathrm{E}\end{array}$ & limestone & $87 \mathrm{~m}$ & flat land \\
\hline Bitti (7) & $\begin{array}{l}40^{\circ} 28^{\prime} 26.0^{\prime \prime} \mathrm{N} \\
09^{\circ} 22^{\prime} 27.4^{\prime \prime} \mathrm{E}\end{array}$ & Granit & $740 \mathrm{~m}$ & mountain \\
\hline Burcei (8) & $\begin{array}{l}39^{\circ} 20^{\prime} 56.8^{\prime \prime} \mathrm{N} \\
09^{\circ} 21^{\prime} 38.7^{\prime \prime} \mathrm{E}\end{array}$ & schists & $530 \mathrm{~m}$ & hill \\
\hline \multicolumn{5}{|c|}{ Collecting stations for Spain-Canary Islands B. bituminosa var. albomarginata plants } \\
\hline Caleta de Famara (9) & $\begin{array}{l}29^{\circ} 06^{\prime} 47.7^{\prime \prime} \mathrm{N} \\
13^{\circ} 33^{\prime} 20.5^{\prime \prime} \mathrm{W}\end{array}$ & $\begin{array}{c}\text { Fisures, gravel } \\
\text { slopes }\end{array}$ & $30 \mathrm{~m}$ & flat land \\
\hline Arecife (10) & $\begin{array}{l}28^{\circ} 59^{\prime} 18.5^{\prime \prime} \mathrm{N} \\
13^{\circ} 32^{\prime} 07.1^{\prime \prime} \mathrm{W}\end{array}$ & Road margins & $54 \mathrm{~m}$ & flat land \\
\hline \multicolumn{5}{|c|}{ Collecting stations for Spain-Canary Islands for B. bituminosa var. crassiuscula plants } \\
\hline $\begin{array}{l}\text { San Cristòbal de la } \\
\text { laguna (11) }\end{array}$ & $\begin{array}{l}28^{\circ} 28^{\prime} 59.8^{\prime \prime} \mathrm{N} \\
16^{\circ} 18^{\prime} 00.0^{\prime \prime} \mathrm{W}\end{array}$ & Rocks, gravel slopes & $503 \mathrm{~m}$ & hill \\
\hline Vilaflor (12) & $\begin{array}{l}28^{\circ} 09^{\prime} 32.5^{\prime \prime} \mathrm{N} \\
16^{\circ} 37^{\prime} 56.6^{\prime \prime} \mathrm{W}\end{array}$ & Rocks, gravel slopes & $1400 \mathrm{~m}$ & mountain \\
\hline \multicolumn{5}{|c|}{ Collecting stations for Spain-Canary Islands for B. bituminosa var. bituminosa plants } \\
\hline $\begin{array}{l}\text { Puntas de } \\
\text { Calnegre (13) }\end{array}$ & $\begin{array}{l}37^{\circ} 30^{\prime} 29.7^{\prime \prime} \mathrm{N} \\
01^{\circ} 24^{\prime} 09.2^{\prime \prime} \mathrm{W}\end{array}$ & Siliceous, ruderal & $25 \mathrm{~m}$ & flat land \\
\hline Llano del Beal (14) & $\begin{array}{l}37^{\circ} 37^{\prime} 29.1^{\prime \prime} \mathrm{N} \\
00^{\circ} 50^{\prime} 29.6^{\prime \prime} \mathrm{W}\end{array}$ & $\begin{array}{l}\text { Nitrified road } \\
\text { margins }\end{array}$ & $105 \mathrm{~m}$ & flat land \\
\hline & (1) & Rocks, gravel slopes & $503 \mathrm{~m}$ & hill \\
\hline
\end{tabular}

The species located in Canary Islands showed a large diversity, with three botanical varieties found in habitats ranging from the coastal semiarid areas on Lanzarote Island with an annual rainfall of $150-300 \mathrm{~mm}(B . b$. var. albomarginata) to the high elevation subhumid area $(1700-2200 \mathrm{~m}, 500 \mathrm{~mm})$ of Tenerife (B. b. var. crassiuscula). The third (B. $b$. var. bituminosa) displayed a wide adaptation across the Canary Islands (300-1000 $\mathrm{m}$ ) and is the only one present in the Mediterranean basin. In the Iberian Peninsula, it has been found in environments ranging from $250-1000 \mathrm{~mm}$ of rainfall and up to $1250-1500 \mathrm{~m}$ of altitude [48]. Germplasms of Bituminaria species and varieties were collected in different Spanish areas (Table 4). The experiments were carried out in plots with 12 plants per accession in a completely randomized design with three replicates. Plants were grown from scarified seeds sown in jiffy pots in a greenhouse and then transplanted to the field in February. Fertilization was done before planting with $46 \mathrm{~kg} \cdot \mathrm{ha}{ }^{-1}$ of $\mathrm{P}_{2} \mathrm{O}_{5}$. Occasional irrigation was supplied to plants, when necessary, from late spring to early summer in the first year.

\subsection{Essential Oil Extraction}

Plant material (200 $\mathrm{g}$ of aerial part) of 15 accessions of Bituminaria genus was submitted to hydrodistillation for $4 \mathrm{~h}$ using a Clevenger-type apparatus. The oils were collected by 
separation from the aqueous phase, dried over anhydrous $\mathrm{Na}_{2} \mathrm{SO}_{4}$, and stored at $-20{ }^{\circ} \mathrm{C}$ before being analyzed. The reached yields $(w / w)$ are reported in Figure 5.

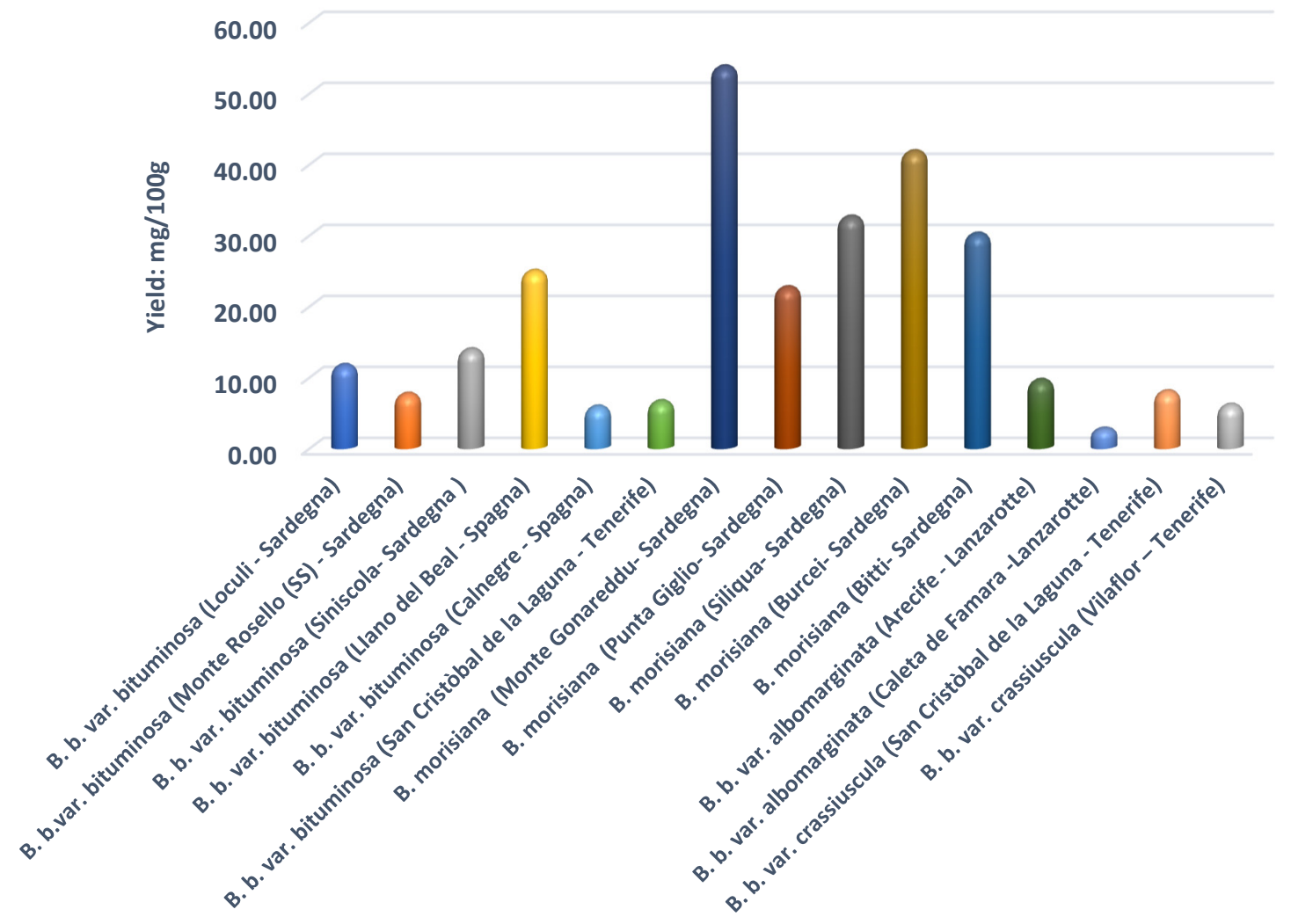

Figure 5. Essential oil yield (mg/100 g) of Bituminaria species and varieties.

\subsection{Gas Chromatography and Mass Spectrometry (GC/MS) Analysis}

Three replicates of the essential oils were separately analyzed using a GC (HewlettPackard Model 5890A, Agilent Technologies Italia S.p.A, Milano, Italy) equipped with a flame ionization detector and fitted with a $60 \mathrm{~m} \times 0.25 \mathrm{~mm}$, thickness $0.25 \mu \mathrm{m}$ ZB- 5 fused silica capillary column (Phenomenex). The injection port and detector temperatures were $280^{\circ} \mathrm{C}$. The column temperature was programmed from $50^{\circ} \mathrm{C}$ to $135^{\circ} \mathrm{C}$ at $5^{\circ} \mathrm{C} / \mathrm{min}(1 \mathrm{~min})$, $5^{\circ} \mathrm{C} / \mathrm{min}$ up $225^{\circ} \mathrm{C}(5 \mathrm{~min}), 5^{\circ} \mathrm{C} / \mathrm{min}$ up $260^{\circ} \mathrm{C}$, and held for $10 \mathrm{~min}$. Oil samples of $0.2 \mu \mathrm{L}$ injection volume were analyzed, diluted in hexane using 2,6-dimethylphenol as an internal standard. Injection was performed using a split/splitless (used in split mode, ratio 50:1) automatic injector HP 7673 and helium as a carrier gas. Several measurements of peak areas were performed through an HP workstation with a threshold set to 0.00 and peak width set to 0.02. Compound quantification was expressed as absolute weight percentage using internal standard ( $n$-tetradecane) response factors (RFs). Since oxygenated compounds have lower detectability than hydrocarbons by FID, detector RFs were determined for key components relative to 2,6-dimethylphenol and assigned to other components on the basis of functional group and/or structural similarity. MS analyses were carried out with an Agilent Technologies model 7820A connected to an MS detector 5977E MSD (Agilent), using the same conditions and column described above. The column was related to the ion source of the mass spectrometer. Mass units were monitored from 10 to $900 \mathrm{at} 70 \mathrm{eV}$. The identification of compounds was based on a comparison of their retention times with those of authentic samples and/or by comparison of their mass spectra with those of published data $[22,49,50]$. 


\subsection{Methanolic Extracts}

The vegetable biomass deriving from the various accessions was cold extracted using methanol acidified with $0.1 \% \mathrm{HCl}(37 \%)$. The fresh aerial plant parts $(100 \mathrm{~g})$ were treated with a grinder and then subjected to maceration three times with $300 \mathrm{~mL}$ of acidified $\mathrm{CH}_{3} \mathrm{OH}$ at room temperature in a flask with a magnetic stir bar at $400 \mathrm{rpm}(30 \mathrm{~h})$. At the end of this period, the resulting solution was dried by evaporating the solvent under vacuum, taking care that the temperature of the water bath did not exceed $50^{\circ} \mathrm{C}$. The residue was dissolved with $250 \mathrm{~mL}$ of distilled water and extracted three times with portions of $100 \mathrm{~mL}$ of $\mathrm{CHCl}_{3}$. The solvent was evaporated under vacuum at room temperature, and the residue was used for subsequent analyzes. The yields of extracts varied between $1.5 \%$ and $2.4 \%$ (Table 5).

Table 5. Yield of methanolic crude extracts of Bituminaria varieties.

\begin{tabular}{lc}
\hline \multicolumn{1}{c}{ Bituminaria Varieties (Station) } & Yield: g/100 g; (Std. Error) \\
\hline Bituminaria bituminosa var. bituminosa (Loculi-Sardegna) & $1.70(0.06)$ \\
Bituminaria bituminosa var. bituminosa (Monte Rosello (SS)_Sardegna) & $1.60(0.06)$ \\
Bituminaria bituminosa var. bituminosa (Siniscola_Sardegna) & $2.00(0.12)$ \\
Bituminaria bituminosa var. bituminosa (LIano del Beal_Spagna) & $1.82(0.09)$ \\
Bituminaria bituminosa var. bituminosa (Calnegre-Spagna) & $2.40(0.12)$ \\
Bituminaria bituminosa var. bituminosa (San Cristòbal de la Laguna-Tenerife) & $2.20(0.06)$ \\
Bituminaria morisiana (Monte Gonareddu-Sardegna) & $2.18(0.07)$ \\
Bituminaria morisiana (Punta Giglio-Sardegna) & $1.90(0.04)$ \\
Bituminaria morisiana (Siliqua-Sardegna) & $1.98(0.07)$ \\
Bituminaria morisiana (Burcei-Sardegna) & $1.52(0.10)$ \\
Bituminaria morisiana (Bitti-Sardegna) & $1.78(0.12)$ \\
Bituminaria bituminosa var. albomarginata (Arecife-Lanzarotte) & $2.00(0.12)$ \\
Bituminaria bituminosa var. albomarginata (Caleta de Famara -Lanzarotte) & $1.91(0.04)$ \\
Bituminaria bituminosa var. crassiuscula (San Cristòbal de la Laguna-Tenerife) & $2.08(0.05)$ \\
Bituminaria bituminosa var. crassiuscula (Vilaflor-Tenerife) & $1.90(0.03)$ \\
\hline
\end{tabular}

\subsection{GC/MS Analyses}

MS analyses were carried out on the extracts (Section 4.5) according to Peroutka et al. [44] with some modification. Briefly, we used an Agilent Technologies model 7820A connected with an MS detector 5977E MSD (Agilent Technologies Italia S.p.A, Milano, Italy), along with a $60 \mathrm{~m} \times 0.25 \mathrm{~mm}$, thickness $0.25 \mu \mathrm{m}$ ZB- $5 \mathrm{~ms}$ fused silica capillary column (Phenomenex). The injection port and detector temperatures were $280^{\circ} \mathrm{C}$. The samples $(0.1 \mu \mathrm{L}$ each) were injected using a split/splitless automatic injector HP 7673 with helium $(1.0 \mathrm{~mL} / \mathrm{min})$ as a carrier gas. Temperature program conditions were as follows: the initial temperature was set at $70^{\circ} \mathrm{C}$, ramped up to $230^{\circ} \mathrm{C}$ at $20^{\circ} \mathrm{C} / \mathrm{min}$, and then ramped up to $250{ }^{\circ} \mathrm{C}$ at $5{ }^{\circ} \mathrm{C} / \mathrm{min}$. The column was related to the ion source of the mass spectrometer. Mass units were monitored from 10 to 900 at $70 \mathrm{eV}$. The quantification of furocoumarins was conducted using the addition method [51] with the necessary variations, in almost all areas of chemical analyses. To avoid excessive errors, the added analyte concentrations were of the same magnitude as those already present in the samples. To not substantially change the solution, we started from a very concentrated standard solution to add minimum volumes. The study of the corresponding variation of the signal obtained allowed us to determine the concentration in the sample, expressed as $\mathrm{mg} / 100 \mathrm{~g}$ of fresh plant (Figure 1).

\subsection{NMR Analysis}

Psoralens were isolated as described in the literature [7] from the extract of B. b. var bituminosa (accession Monte Rosello, SS) and from the extract of B. morisiana (accession Monte Gonareddu).

One gram of the crude extract of $B . b$. var. bituminosa derived from the Monte Rosello accession was suspended in $n$-hexane and placed at the top of a silica gel column $(20 \mathrm{~mm}$ 
$\times 2.5 \mathrm{~mm}$, Kieselgel $60,0.015-0.040 \mathrm{~mm}$ ), before eluting with $n$-hexane $/ \mathrm{Et}_{2} \mathrm{O}$ at different ratios with increasing $\mathrm{Et}_{2} \mathrm{O}$ concentration. All collected fractions were analyzed by TLC, by elution with $n$-hexane/ $\mathrm{Et}_{2} \mathrm{O}(1: 1 \mathrm{v} / \mathrm{v})$. Pure fractions containing angelicin $(40 \mathrm{mg})$ and psoralen $(5 \mathrm{mg})$ were obtained.

The same procedure was applied to $1 \mathrm{~g}$ of crude extract of $B$. morisiana derived from the Monte Gonareddu accession; in this case, we obtained $8 \mathrm{mg}$ of angelicin and $19 \mathrm{mg}$ of psoralen.

The identity of these compounds was assessed by ${ }^{1} \mathrm{H}$ and ${ }^{13} \mathrm{C}$ nuclear magnetic resonance (NMR), using a VARIAN (Mercury plus) spectrometer operating at $399.93 \mathrm{MHz}$ for ${ }^{1} \mathrm{H}$ and $100.57 \mathrm{MHz}$ for ${ }^{13} \mathrm{C}$, with the sample dissolved in $\mathrm{CDCl}_{3}$, using tetramethylsilane (TMS) as an internal reference.

Angelicin: ${ }^{1} \mathrm{H}-\mathrm{NMR}\left(400 \mathrm{MHz}, \mathrm{CHCl}_{3}\right), \delta$ ppm: $7.83(1 \mathrm{H}, d, \mathrm{~J}=9.6 \mathrm{~Hz}) ; 7.71(1 \mathrm{H}, \mathrm{d}$, $\mathrm{J}=2.4 \mathrm{~Hz}) ; 7.45(1 \mathrm{H}, d, \mathrm{~J}=8.8 \mathrm{~Hz}) ; 7.40(1 \mathrm{H}, d, \mathrm{~J}=8.8 \mathrm{~Hz}) ; 7.15(1 \mathrm{H}, d, \mathrm{~J}=2.4 \mathrm{~Hz}) ; 6.41$ $(\mathrm{H}, d, \mathrm{~J}=9.6 \mathrm{~Hz}) .{ }^{13} \mathrm{C}-\mathrm{NMR}\left(100 \mathrm{MHz}, \mathrm{CHCl}_{3}\right), \delta$ ppm: 160.66 (C-2), 157.33 (C-7), 148.93 (C-9), 145.90 (C-12), 144.59 (C-4), 141.25 (C-3), 123.82 (C-5), 119.82 (C-5), 117.04 (C-8), 114.10 (C-10), 108.84 (C-6), 104.11 (C-11).

Psolaren: ${ }^{1} \mathrm{H}-\mathrm{NMR}\left(400 \mathrm{MHz}, \mathrm{CHCl}_{3}\right), \delta$ ppm: $7.82(1 \mathrm{H}, d, \mathrm{~J}=10 \mathrm{~Hz}) ; 7.71(1 \mathrm{H}, d$, $\mathrm{J}=2.4 \mathrm{~Hz}) ; 7.70(1 \mathrm{H}, \mathrm{s}) ; 6.84(1 \mathrm{H}, d, \mathrm{~J}=2.4 \mathrm{~Hz}) ; 6.39(\mathrm{H}, d, \mathrm{~J}=10 \mathrm{~Hz}) .{ }^{13} \mathrm{C}-\mathrm{NMR}(100 \mathrm{MHz}$, $\mathrm{CHCl}_{3}$ ), $\delta$ ppm: 161.10 (C-2), 156.37 (C-7), 151.96 (C-9), 146.91 (C-12), 144.12 (C-4), 124.86 (C-6), 119.82 (C-5), 115.37 (C-10), 114.60 (C-3), 106.35 (C-11), 99.87 (C-8).

\subsection{Statistical Analysis}

Data analyses of three replicates were performed for every sample. ANOVA was applied with a factorial design (MSTAT-C, software developed by the Crop and Soil Sciences Department of Michigan State University of the United States). Mean separation was tested by application of Tukey's test. To investigate chemical variation in the 15 different accessions of Bituminaria based on GC and GC/MS, we submitted the data to multivariate statistical evaluation (PCA). PCA is an unsupervised pattern recognition technique that converts data consisting of many interrelated variables to a new coordinate system, thereby reducing dimensionality while maintaining the variance [52]. PCA reveals trends in a dataset such as groupings and clusters based on chemical similarities or differences, while outliers within the dataset are also identified. The results of PCA were observed in a score scatter plot, displaying the spatial distribution of observations. Prior to chemometric analysis, the total integral areas were set to 100 to normalize the data, and the generated ASCII file was imported into Microsoft Excel for labeling. The matrix was then imported into SIMCA-P software version 12.0 (Umetrics AB, Umea, Sweden) for statistical analysis.

\section{Conclusions}

The aim of the present research was to discriminate Bituminaria populations with low furocoumarin content, useful for fodder production in Mediterranean environments, or Bituminaria populations with high furocoumarin content, useful for pharmaceutical purposes. For this reason, we characterized the volatile compounds of 15 different accessions of native Sardinian populations of B. morisiana and B. bituminosa and Spanish native accessions of $B$. bituminosa and its varieties. We demonstrated that it is not easy recognize the various plants belonging to the genus Bituminaria using secondary metabolites, because most represented constituents are often the same (although in different concentrations); however, considering all characterized volatile compounds in our work and carrying out a principal component analysis, it was possible to clearly distinguish the species $B$. morisiana and $B$. bituminosa. Moreover, using only components of the essential oils, we also evidenced the differences between $B . b$. var. crassiuscula and the other B. bituminosa varieties, as well as $B$. morisiana, as reported in the score plot of PCA analysis performed using the essential oil components, where a clear differentiation of the varieties $B . b$. var. crassiuscula and B. morisiana from B. b. var. bituminosa and B. b. var. Albomarginata is shown. 
Author Contributions: C.P. and R.A.M.M. handled the germplasm, conducted biomorphological screening, and took care of all agronomic factors, seed production, and its components; conceptualization, C.P.; analytic methodology, interpretation of data, and conceptualization, M.U.; statistical analysis, nuclear magnetic resonance analysis and interpretation of data, M.M.; writing-original draft preparation, M.U. and M.M. All authors have read and agreed to the published version of the manuscript.

Funding: This research was funded by the Sardinian Government, L.R. n.7/2007 and by FAR2019USAIUniversity of Sassari (Special Grant “Una Tantum 2019”).

Institutional Review Board Statement: Not applicable.

Informed Consent Statement: Not applicable.

Conflicts of Interest: The authors declare no conflict of interest.

Sample Availability: Samples of the compounds are not available from the authors.

\section{References}

1. Murray Vail, A. A Study of the Genus Bituminaria in America. Bull. Torrey Bot. Club 1894, 21, 91-119. [CrossRef]

2. Brullo, S.; Brullo, C.; Cambria, S.; Cristaudo, A.; Giusso del Galdo, G. Bituminaria antiatlantica (Psoraleeae, Fabaceae), a new species from Morocco. PhytoKeys 2017, 85, 109-124. [CrossRef] [PubMed]

3. Muñoz, A.; Ortiz-Dorda, J.; Correal, E. Morphological and molecular characterization of Biturninaria bituminosa accessions from South-East Spain and the Canary Islands. Cahiers Options Méditerranéennes 2000, 45, 103-107.

4. Camarda, I. Le Piante endemiche della Sardegna: 91. Boll. Della Soc. Sarda Di Sci. Nat. 1981, 20, $269-274$.

5. Méndez, P.; Fernández, M.; Santos, A. Variedades de Bituminaria bituminosa (L.) Stirt. (Leguminosae) en el archipiélago canario. Pastos 1990, 20-21, 157-166.

6. Real, D.; Correal, E.; Méndez, P.; Santos, A.; Ríos Ruiz, S.; Sternberg, M.; Dini-Papanastasi, O.; Pecetti, L.; Tava, A. Bituminaria bituminosa C.H. Stirton (synonym: Psoralea bituminosa L.). In 'Grassland Species'; FAO: Rome, Italy. Available online: http: //www.fao.org/ag/agp/AGPC/doc/Gbase/new_species/tedera/bitbit.htm (accessed on 15 March 2019).

7. Pecetti, L.; Tava, A.; Pagnotta, M.A.; Russi, L. Variation in forage quality and chemical composition among Italian accessions of Bituminaria bituminosa (L.) Stirt. J. Sci. Food Agric. 2007, 87, 985-991. [CrossRef]

8. Viola, G.; Salvador, A.; Vedaldi, D.; Dall'Acqua, F.; Bianchi, N.; Zuccato, C.; Borgatti, M.; Lampronti, I.; Gampari, R. Differentiation and apoptosis in UVA-irradiated cells treated with furocoumarin derivatives. Ann. N. Y. Acad. Sci. 2009, 1171, 334-344. [CrossRef]

9. Sah, P.; Agarwal, D.; Garg, S.P. Isolation and identification of furocoumarins from the seeds of Psoralea corylifolia Linn. Indian J. Pharm. Sci. 2006, 68, 768-771. [CrossRef]

10. Lu, Y.; An, J.; Jiang, Y. Rapid analysis of psoralen and isopsoralen in Psoralea corylifolia by microwave-assisted extraction. Zhongguo Zhong Yao Za 2011, 36, 1158-1161.

11. Parast, B.M.; Rasouli, M.; Manafi, M.; Agrawal, V. Quantification of psoralen in plant parts of Psoralea Corylifolia growing in vivo and in vitro and enhancement of psoralen by organic elicitors. Anal. Chem. Lett. 2012, 2, 227-234. [CrossRef]

12. Jeong, M.; Hong, T.; Lee, K.; Hwangbo, H.; Kim, M.; Ma, W.; Zahn, M. HPLC method for simultaneous quantification of bakuchiol and minor furocoumarins in bakuchiol extract from Psoralea corylifolia. J. AOAC Int. 2015, 98, 902-906. [CrossRef]

13. Del Río, J.A.; Ortuño, A.; Pérez, I.; Bennett, R.G.; Real, D.; Correal, E. Furanocoumarin content in Bituminaria bituminosa varieties and Cullen species. In The Contributions of Grasslands to the Conservation of Mediterranean Biodiversity; Porqueddu, C., Ríos, S., Eds.; The Food and Agriculture Organization: Zaragoza, Spain, 2010; pp. 69-70.

14. Pecetti, L.; Mella, M.; Tava, A. Variation in herbage biochemical composition among pitch trefoil (Bituminaria bituminosa) populations from Elba Island, Italy. J. Agric. Food Chem. 2016, 64, 195-203. [CrossRef]

15. Innocenti, G.; Cappelletti, E.M.; Caporale, G. Furocoumarin contents in the vegetative organs of cultivated Psoralea species. Int. J. Pharmacogn. 1991, 29, 311-316. [CrossRef]

16. Innocenti, G.; Piovan, A.; Filippini, R.; Caniato, R.; Cappelletti, E.M. Quantitative recovery of furanocoumarins from Psoralea bituminosa. Phytochem. Anal. 1997, 8, 84-86. [CrossRef]

17. Bandeira Reidel, R.V.; Giovanelli, S.; Pipitone, A.; Minissale, P.; Pistelli, L. Phytochemical study of Bituminaria basaltica aerial parts, an Italian endemism. Nat. Prod. Res. 2017, 31, 2375-2380. [CrossRef] [PubMed]

18. Bondarenko, A.S.; Aizenman, B.E.; Zatula, D.G. Extraction from Psoralea drupacea Bge. of bakuchiol and its antimicrobial activity. Tr. S'ezda Mikrobiol. Ukr 1975, 4, 208-209.

19. Bondarenko, A.S.; Aizenman, B.E.; Prikhod'ko, V.A.; Meshcheryakov, A.A.; Skorobogat'ko, T.I. Antibiotic properties of the essential oil of Bituminaria drupacea. Mikrobiol. Zhur. 1973, 34, 612-616.

20. Zutshi, S.K.; Bhagwat, A.W. Effect of the essential oil of Bituminaria corylifolia (Linn.) (P.C.) on isolated rectus abdominis of frog (Rana tigrina). Indian J. Physiol. Pharm. 1977, 21, 165-166.

21. Bertoli, A.; Menichini, F.; Noccioli, C.; Morelli, I.; Pistelli, L. Volatile constituents of different organs of Bituminaria bituminosa L. Flavour Fragr. J. 2004, 19, 166-171. [CrossRef] 
22. Tava, A.; Pecetti, L.; Ricci, M.; Pagnotta, M.A.; Russi, L. Volatile compounds from leaves and flowers of Bituminaria bituminosa (L.) Stirt. (Fabaceae) from Italy. Flavour Fragr. J. 2007, 22, 363-370. [CrossRef]

23. El-Seedi, H.; Zayed, M.; Roshdy, S.; Salem, M.; Hawata, M.; El-Essawy, F.; El-Barbary, M.; El-Kousy, S. Analysis of the essential oil from the aerial parts of Psoralea pubescence (Miq.) Standl and its antibacterial activity. Med. Chem. Res. 2010, 19, 1036-1042. [CrossRef]

24. Pistelli, L.; Noccioli, C.; Appendino, G.; Bianchi, F.; Sterner, O.; Ballero, M. Pterocarpans from Bituminaria morisiana and Bituminaria bituminosa. Phytochemistry 2003, 64, 595-598. [CrossRef]

25. Cottiglia, F.; Casu, L.; Bonsignore, L.; Casu, M.; Floris, C.; Leonti, M.; Gertsch, J.; Heilmann, J. New cytotoxic prenylated isoflavonoids from Bituminaria morisiana. Planta Med. 2005, 71, 254-260. [CrossRef] [PubMed]

26. Porqueddu, C.; Melis, R.A.M.; Re, G.A.; Usai, M.; Marchetti, M. Forage production and essential oil content of Bituminaria bituminosa and P. morisiana accessions. The Role of Grasslands in a Green Future. Threats and Perspectives in Less Favoured Areas. In Proceedings of the 17th Symposium of the European Grassland Federation, Akureyri, Iceland, 23-26 June 2013; Helgadóttir, Á., Hopkins, A., Eds.; Agricultural University of Iceland: Borgarnes, Iceland, 2013; Volume 18, pp. 349-351.

27. Melis, R.A.M.; Franca, A.; Re, G.A.; Porqueddu, C. Bio-agronomic characterization and implications on the potential use as forage of Bituminaria bituminosa and B. morisiana accessions. Grass Forage Sci. 2018, 73, 284-296. [CrossRef]

28. Iannuzzi, A.; Perucatti, A.; Genualdo, V.; Pauciullo, A.; Melis, R.; Porqueddu, C.; Marchetti, M.; Usai, M.; Iannuzzi, L. Sister chromatid exchange (SCE) test in river buffalo lymphocytes treated in vitro with furocoumarin extracts. Mutagenesis 2016, 31, 547-551. [CrossRef]

29. Boukhris, M.; Bouaziz, M.; Feki, I.; Jemai, H.; El Feki, A.; Sayadi, S. Hypoglycemic and antioxidant effects of leaf essential oil of Pelargonium graveolens L'Her. in alloxan induced diabetic rats. Lipids Health Dis. 2012, 11, 1-10. [CrossRef] [PubMed]

30. Nivinsliene, O.; Butkiene, R.; Gudalevic, A.; Mockute, D.; Meskauskiene, V.; Grigaliunaite, B. Influence of urban environment on chemical composition of Tilia cordata essential oil. Chemija 2007, 18, 44-49.

31. Jian-Yu, S.; Zhu, L.; Tian, Y.-J. Chemical composition and antimicrobial activities of essential oil of Matricaria songarica. Int. J. Agric. Biol. 2012, 14, 107-110.

32. Farsam, H.; Amanlou, M.; Taghi-Cheetsaz, N.; Amin, G.R.; Saledi-Sormaghi, M.H. Essential oil of Chimonanthus fragrans flowers population of Tehran. DARU J. Pharm. Sci. 2007, 15, 129-131.

33. Javidnia, K.; Miri, R.; Kamalinejad, M.; Nasiri, A. Composition of the essential oil of Salvia mirzayanii Rech. f. Esfand from Iran. Flavour Fragr. J. 2002, 17, 465-467. [CrossRef]

34. Morteza-Semnani, K.; Saeedi, M.; Changizi, S. The essential oil composition of Hypericum scabrum L. from Iran. Flavour Fragr. J. 2006, 21, 513-515. [CrossRef]

35. Sylvestre, M.; Pichette, A.; Longtin, A.; Nagau, F.; Legault, J. Essential oil analysis and anticancer activity of leaf essential oil of Croton flavens L. from Guadeloupe. J. Ethnopharmacol. 2006, 103, 99-102. [CrossRef] [PubMed]

36. Malencic, D.J.; Couladis, M.; Mimica-Dukic, N.; Popovic, M.; Boza, P. Essential oils of three Salvia species from the Pannonian part of Serbia. Flavour Fragr. J. 2004, 19, 225-228. [CrossRef]

37. Kristiawan, M.; Sobolik, V.; Al-Haddad, M.; Allaf, K. Effect of pressure-drop rate on the isolation of cananga oil using instantaneous controlled pressure-drop process. Chem. Eng. Process. 2008, 47, 66-75. [CrossRef]

38. Fakhari, A.R.; Sonboli, A.; Heydari, R. Composition of the essential oil of Rhabdosciadium strausii from Iran. Chem. Nat. Compd. 2005, 41, 413-414. [CrossRef]

39. Baranauskiene, R.; Venskutonis, R.P.; Demyttenaere, J.C.R. Sensory and instrumental evaluation of catnip (Nepeta cataria L.) aroma. J. Agric. Food Chem. 2003, 51, 3840-3848. [CrossRef]

40. Senatore, F.; Rigano, D.; de Fusco, R.; Bruno, M. Composition of the essential oil from flowerheads of Chrysanthemum coronarium L. (Asteraceae) growing wild in Southern Italy. Flavour Fragr. J. 2004, 19, 149-152. [CrossRef]

41. Xu, X.; Tang, Z.; Liang, Y. Comparative analysis of plant essential oils by GC-MS coupled with integrated chemometric resolution methods. Anal. Methods 2010, 2, 359-367. [CrossRef]

42. Arctander, S. (Ed.) Perfume and Flavor Chemicals (Aroma Chemicals); Allured Publishing Corporation: Montclair, NJ, USA, 1969; Volume I.

43. Burns, D.A. Rook's Textbook of Dermatology. In Diseases Caused by Arthropods and Other Noxious Animals, 8th ed.; Burns, T., Breathnach, S., Neil Cox, N., Griffiths, C., Eds.; Wiley-Blackwell: Hoboken, NJ, USA, 2013; p. 38.

44. Peroutka, R.; Schulzova, V.; Botek, P.; Hajslova, J. Analysis of furanocoumarins in vegetables (Apiaceae) and citrus fruits (Rutaceae). J. Sci. Food Agric. 2007, 87, 2152-2163. [CrossRef]

45. Amatori, S.; Bagaloni, I.; Macedi, E.; Formica, M.; Giorgi, L.; Fusi, V.; Fanelli Malten, M. A new synthetic molecule showing in vitro antiproliferative activity against tumor cells and induction of complex DNA structural alterations. Br. J. Cancer 2010, 103, 239-248. [CrossRef]

46. Hart, B.A.; Copray, S.; Philippens, I. Apocynin, a low molecular oral treatment for neurodegenerative disease. BioMed Res. Int. 2014, 2014, 298020. [CrossRef] [PubMed]

47. Scheiba, N.; Andrulis, M.; Helmbold, P. Treatment of shiitake dermatitis by balneo PUVA therapy. J. Am. Acad. Dermatol. 2011, 65, 453-455. [CrossRef] [PubMed] 
48. Correal, E.; Hoyos, A.; Ríos, S.; Méndez, P.; Real, D.; Snowball, R.; Costa, J. Seed production of Bituminaria bituminosa: Size, production, retention and germination capacity of the legumes. In Sustainable Mediterranean Grasslands and Their Multi-Functions; Porqueddu, C., Tavares de Sousa, M.M., Eds.; CIHEAM/FAO/ENMP/SPPFOptions Méditerranéennes: Série A. Séminaires Méditerranéens; n. 792008 pag; Sassari: Zaragoza, Spain, 2008; pp. 379-383.

49. NIST2011. Library of Mass Spectra; Agilent Technologies Co.: Palo Alto, CA, USA, 2011.

50. Adams, R.P. Identification of Essential Oil Components by Gas Chromatography/Mass Spectrometry, 4th ed.; Allured Publ. Corp.: Carol Stream, IL, USA, 2007.

51. Saxberg Bo, E.H.; Kowalski, B.R. Generalized standard addition method. Anal. Chem. 1979, 51, 1031-1038. [CrossRef]

52. Bansal, A.; Chhabra, V.; Rawal, K.; Sharma, S. Chemometrics: A new scenario in herbal drug standardization. J. Pharm. Anal. 2014, 4, 223-233. [CrossRef] 